Restrições aos conjuntos de rotação

dos geradores de grupos Abelianos

de homeomorfismos de $\mathbb{T}^{2}$

\author{
Deissy Milena Sotelo Castelblanco
}

\author{
TESE APRESENTADA \\ $\mathrm{AO}$ \\ Instituto De Matemática e Estatística \\ DA \\ Universidade DE SÃO PAUlo \\ PARA \\ OBTENÇÃO DO TÍTULO \\ $\mathrm{DE}$ \\ DOUTOR EM CIÊNCIAS \\ Programa: Matemática Aplicada \\ Orientador: Prof. Dr. Fábio Armando Tal
}

Durante o desenvolvimento deste trabalho a autora recebeu auxílio financeiro da CAPES

São Paulo, julho de 2015 


\title{
Restrições aos conjuntos de rotação dos geradores de grupos Abelianos de homeomorfismos de $\mathbb{T}^{2}$
}

\author{
Esta versão da tese contém as correções e alterações sugeridas \\ pela Comissão Julgadora durante a defesa da versão original do trabalho, \\ realizada em 16/06/2015. Uma cópia da versão original está disponível no \\ Instituto de Matemática e Estatística da Universidade de São Paulo.
}

Comissão Julgadora:

- Prof. Dr. Fábio Armando Tal (orientador) - IME-USP

- Prof. Dr. Salvador Addas-Zanata - IME-USP

- Prof. Dr. Ricardo dos Santos Freire Junior - IME-USP

- Prof. Dr. Alejandro Kocsard - UFF

- Prof. Dr. Mauro Benayon Menezes - UFRRJ 
A mis Padres 
A fé é a certeza daquilo que esperamos e a prova das coisas que não vemos.

Hebreus 11:1

Nuestra recompensa se encuentra en el esfuerzo y no en el resultado. Un esfuerzo total es una victoria completa. Mahatma Gandhi 


\section{Agradecimentos}

Graças a Deus por tudo!

Agradeço ao IME por dar-me a oportunidade de fazer parte dessa comunidade; ao meu orientador Fábio por sua dedicação, pela paciência, por acreditar em mim e pelas interessantes conversas.

À tese do Mauro que me serviu de inspiração. À Google pelas valiosas informações encontradas até agora.

À minha mãe pela força e motivação que sempre me dá e me ajudou para continuar. À minha família pelo apoio. Aos amigos do IME pela companhia, em especial à Gleiciane pelas valiosas sugestões na escrita da tese e à Nubia pelos seus conselhos.

À Professora Beatriz Villa por seus ensinamentos e as palavras que me incentivaram para seguir os meus estudos de pós-graduação.

À CAPES pelo auxílio financeiro e à USP em geral por todos os benefícios recebidos como estudante. 


\section{Resumo}

CASTELBLANCO, D. M. S. Restrições aos conjuntos de rotação dos geradores de grupos Abelianos de homeomorfismos de $\mathbb{T}^{2}$. 2015. 61 p. Tese (Doutorado) Instituto de Matemática e Estatística, Universidade de São Paulo, São Paulo, 2015.

Dados dois conjuntos compactos e convexos $K_{1}, K_{2} \subset \mathbb{R}^{2}$, queremos saber se existem $f$ e $h$, dois homeomorfismos de $\mathbb{T}^{2}$, homotópicos à identidade, que comutam, com levantamentos $\tilde{f}$ e $\tilde{h}$, tais que $K_{1}$ e $K_{2}$ são os seus conjuntos de rotação, respectivamente. Neste trabalho, mostramos alguns casos onde isto não pode acontecer, assumindo restrições nos conjuntos de rotação. Além disso, introduzimos o conceito de conjunto de rotação para semigrupos Abelianos finitamente gerados por homeomorfismos homotópicos à identidade, mostrando um caso em que o semigrupo é anular.

Palavras-chave: Homeomorfismos do toro, conjunto de rotação, semigrupo Abeliano. 


\begin{abstract}
CASTELBLANCO, D. M. S. Restrictions on rotation sets of generators of Abelian groups of homeomorphisms of $\mathbb{T}^{2} 2015$. 61 p. These (Doctoral) - Instituto de Matemática e Estatística, Universidade de São Paulo, São Paulo, 2015.

Let $K_{1}, K_{2} \subset \mathbb{R}^{2}$ be two convex, compact sets. We would like to know if there are commuting homeomorphisms $f$ and $h$ of $\mathbb{T}^{2}$, homotopic to the identity, with lifts $\tilde{f}$ and $\tilde{h}$, such that $K_{1}$ and $K_{2}$ are their rotation sets, respectively. In this work, we proof some cases where it cannot happen, assuming some restrictions on rotation sets. Besides that, we introduce the concept of rotation set for Abelian semi-groups finitely generated by homeomorphisms homotopic to the identity, showing a case where the semi-group is annular.
\end{abstract}

Keywords: Torus homeomorphisms, rotation set, Abelian semi-group. 


\section{Conteúdo}

1 Introdução 1

1.1 Considerações preliminares $\ldots \ldots \ldots \ldots \ldots \ldots$

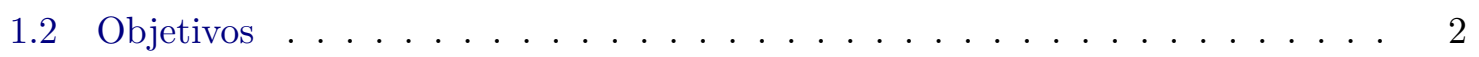

1.3 Organização do trabalho . . . . . . . . . . . . . . . . . . . . 4

2 Preliminares $\quad \mathbf{5}$

2.1 Número de rotação para homeomorfismos de $\mathbb{S}^{1} \ldots \ldots \ldots \ldots$

2.2 Conjunto de rotação em variedades compactas . . . . . . . . . . . . . 6

2.3 Conjunto de rotação no toro . . . . . . . . . . . . . . . . . . . . . 10

2.4 Propriedades do conjunto de rotação . . . . . . . . . . . . . . . . . . . . . 12

2.5 Homeomorfismo anular . . . . . . . . . . . . . . . . . . . . . . . . 21

2.6 Lema de Atkinson . . . . . . . . . . . . . . . . . . . . . . . . . 24

3 Grupo Abeliano de homeomorfismos de $\mathbb{T}^{2} \quad \mathbf{2 5}$

3.1 Conjunto de rotação do semigrupo . . . . . . . . . . . . . . . 25

3.2 Grupo anular . . . . . . . . . . . . . . . . . . . . . . . . 31

3.3 Resultados recentes . . . . . . . . . . . . . . . . . . . . . . . . 31

4 Restrições aos conjuntos de rotação $\quad 33$

4.1 Motivação do problema . . . . . . . . . . . . . . . . . . . 33

4.2 Demonstração do teorema . . . . . . . . . . . . . . . . . . . . 36

4.2 .1 Resultado principal . . . . . . . . . . . . . . . . . . . . 39

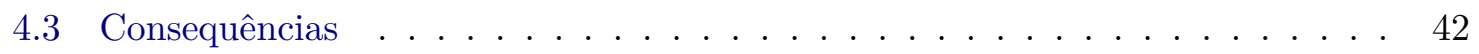

$\begin{array}{ll}\text { Bibliografia } & 45\end{array}$ 
CONTEÚDO 


\section{Capítulo 1}

\section{Introdução}

Neste trabalho estudamos o conjunto de rotação de homeomorfismos do toro $T^{2}=\mathbb{R}^{2} / \mathbb{Z}^{2}$, homotópicos à identidade que comutam.

\subsection{Considerações preliminares}

A definição de conjunto de rotação de homeomorfismos do toro, foi introduzida por M. Misiurewicz e K. Ziemian [MZ89], como uma generalização do conceito de número de rotação de um homeomorfismo $h$ que preserva orientação no círculo $\mathbb{S}^{1}=\mathbb{R} / \mathbb{Z}$. Este último foi introduzido por H. Poincaré para estudar a dinâmica dessas aplicações, e é um dos conceitos mais conhecidos e estudados em sistemas dinâmicos. Os resultados de Poincaré [Poi52] e Denjoy [Den32] mostram que muitas propriedades da dinâmica são caracterizadas pelo número de rotação.

Consideremos um homeomorfismo $f$ do toro, homotópico à identidade e denotemos por $\tilde{f}$ um levantamento de $f$ ao recobrimento universal $\mathbb{R}^{2}$. O conjunto de rotação no toro $\mathbb{T}^{2}$, denotado por $\rho(\tilde{f})$, tem-se tornado uma ferramenta muito importante, já que possui propriedades, que permitem entender a dinâmica de $f$. Por exemplo, sabemos de [MZ89] que pontos extremais racionais no conjunto de rotação são realizados por órbitas periódicas. E de [LM91] que se $\tilde{f}$ tem conjunto de rotação com interior não vazio, então $f$ tem entropia topológica positiva.

No estudo do conjunto de rotação, encontramos em [MZ89] que ele é um conjunto compacto e convexo. Logo $\rho(\tilde{f})$ pode ser de três tipos: ou um vetor, ou um segmento de reta ou ter interior não vazio. Dada a importância do conjunto de rotação, aparecem questões relacionadas à sua forma, como por exemplo:

Questão 1. Dado qualquer conjunto compacto e convexo $K \subset \mathbb{R}^{2}$, existe algum homeomorfismo $f$ do toro, homotópico à identidade, tal que $\rho(\tilde{f})=K$ ? 
Esta pergunta só foi respondida neste ano em [CT15], onde P. Le Calvez e F. Tal, mostraram que um segmento de reta com inclinação irracional e um ponto racional no seu interior, não pode ser realizado como um conjunto de rotação de um homeomorfismo do toro, homotópico à identidade.

O caso de um vetor, digamos $K=\{v\}$, sempre pode ser realizado como o conjunto de rotação da rotação rígida $R_{v}$. Quando $K$ é um segmento de reta, sabemos que existem homeomorfismos do toro, onde segmentos de reta com inclinação racional ou segmentos de reta com inclinação irracional e um ponto extremal racional, podem ser o seu conjunto de rotação [FM90]. E um polígono convexo $K$ com vértices racionais, também pode ser realizado como conjunto de rotação [Kwa92].

\subsection{Objetivos}

Nosso primeiro objetivo é definir o conjunto de rotação para um semigrupo abeliano, finitamente gerado por homeomorfismos do toro, homotópicos à identidade e estudar suas propriedades.

Nesta direção, existe a tese [Ben13], sobre grupos abelianos de homeomorfismos irrotacionais, conservativos, onde se generaliza uma classificação dada em [KT14a] para esse tipo de aplicações.

Neste trabalho, também buscaremos estudar a generalização da Questão 1, feita anteriormente para um homeomorfismo homotópico à identidade. Especificamente estamos interessados em responder à seguinte pergunta:

Questão 2. Dados dois conjuntos compactos e convexos $K_{1}, K_{2} \subset \mathbb{R}^{2}$, existem $f$ e $h$, dois homeomorfismos de $\mathbb{T}^{2}$, homotópicos à identidade, que comutam, tais que $K_{1}$ e $K_{2}$ sejam os seus conjuntos de rotação, respectivamente?

Com essa finalidade, nos restringiremos aos conjuntos compactos e convexos que já sabemos, podem ser realizados como conjuntos de rotação. Esses conjuntos são então, por exemplo ou um ponto, ou um segmento de reta de inclinação racional ou um polígono convexo com vértices racionais, como comentamos anteriormente na Seção 1.1 e veremos com mais detalhes no Capítulo 2.

Apresentamos alguns exemplos de pares de conjuntos compactos e convexos $K_{1}, K_{2}$ que respondem afirmativamente à Questão 2, mas o resultado principal desta tese mostra ser negativa a resposta no caso geral.

No seguinte resultado, usamos os conceitos de Deslocamento e Desvio na direção de $v \in \mathbb{R}_{*}^{2}$, definidos como segue:

$$
\operatorname{Desl}_{v}(\tilde{f})=\sup _{\substack{n \in \mathbb{Z} \\ x \in \mathbb{R}^{2}}}\left|\operatorname{Pr}_{v}\left(\tilde{f}^{n}(x)-x\right)\right|
$$




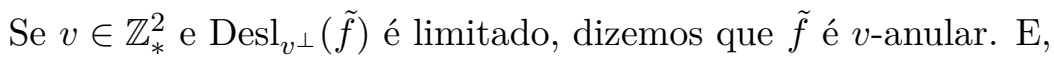

$$
\operatorname{Desv}_{v}(\tilde{f})=\sup _{\substack{n \in \mathbb{Z} \\ x, y \in \mathbb{R}^{2}}}\left|\operatorname{Pr}_{v}\left[\left(\tilde{f}^{n}(x)-x\right)-\left(\tilde{f}^{n}(y)-y\right)\right]\right|,
$$

onde $\operatorname{Pr}_{v}(z)=\frac{\langle z, v\rangle}{\|v\|}$, denota a projeção ortogonal.

Teorema A. Sejam $f, h \in \operatorname{hom}_{0}\left(\mathbb{T}^{2}\right)$ que comutam e $\tilde{f}, \tilde{h}$ respectivos levantamentos. Se $\tilde{f}$ é v-anular, para algum $v \in \mathbb{Z}_{*}^{2}$, então $\operatorname{Desv}_{v^{\perp}}(\tilde{h})<\infty$ ou $\operatorname{Desv}(\tilde{f})<\infty$.

Como consequência deste teorema, temos em particular as seguintes restrições aos conjuntos de rotação de homeomorfismos de $\mathbb{T}^{2}$, que respondem negativamente à Questão 2.

Consideremos $v \in \mathbb{Z}_{*}^{2}$ e a reta $L=\{t v ; t \in \mathbb{R}\}$.

Teorema B. Se $\rho(\tilde{f})$ é um segmento da reta $L$, com pontos racionais e $\rho(\tilde{h})$ é qualquer um dos seguintes casos:

1. Tem interior não vazio.

2. Um segmento de reta não degenerado, numa direção não paralela à $v$.

3. Um ponto contido na reta $L$, sendo $\tilde{h}$ não $v$-anular.

Então, em cada caso $\tilde{f}$ e $\tilde{h}$ não comutam.

Consideremos agora um subgrupo Abeliano $\mathcal{G}$, gerado por $s$ homeomorfismos do toro homotópicos à identidade $f_{1}, \ldots, f_{s}$. Definimos o deslocamento de $\tilde{G}$ na direção $v \in \mathbb{Z}_{*}^{2}$ como,

$$
\operatorname{Desl}_{v}(\tilde{\mathcal{G}})=\sup _{\substack{\tilde{g} \in \mathcal{G} \\ x \in \mathbb{R}^{2}}}\left|\operatorname{Pr}_{v}(\tilde{g}(x)-x)\right| .
$$

Dizemos que $\tilde{\mathcal{G}}$ é $v$-anular, se o deslocamento de $\tilde{\mathcal{G}}$ na direção de $v^{\perp}$ é limitado.

Outro resultado em relação à dinâmica do grupo, conhecidas propriedades dos conjuntos de rotação dos geradores é o seguinte:

Teorema C. Seja $\mathcal{G}$ como acima. Suponhamos que todo gerador $f_{i}$ possui um levantamento $\tilde{f}_{i}$, tal que $0 \in \operatorname{Pr}_{v^{\perp}}\left(\rho\left(\tilde{f}_{i}\right)\right)$, e que existe algum elemento $f \in \mathcal{G}$, tal que $\rho(\tilde{f})$ é um segmento não degenerado da reta $L$. Então, $\mathcal{G}$ é anular. 


\subsection{Organização do trabalho}

Para um melhor acompanhamento na leitura desta tese, organizamos os capítulos da seguinte forma:

No Capítulo 2, introduzimos os conceitos preliminares da teoria de conjunto de rotação e apresentamos alguns resultados sobre homeomorfismos anulares e o Lema de Atkinson, que serão usados no Capítulo 4.

No Capítulo 3, estudamos o semigrupo Abeliano gerado por homeomorfismos do toro, homotópicos à identidade, generalizando a definição de conjunto de rotação para estes semigrupos, e mostramos algumas propriedades preliminares do seu conjunto de rotação.

Finalmente no Capítulo 4, fazemos a demonstração do resultado principal e discutimos suas consequências. 


\section{Capítulo 2}

\section{Preliminares}

Neste capítulo vamos introduzir a teoria que usaremos no Capítulo 4, nas demonstrações dos teoremas. Enunciamos alguns conceitos preliminares da teoria de conjunto de rotação existente, e estudamos algumas propriedades do conjunto de rotação no toro e o Lema de Atkinson.

\subsection{Número de rotação para homeomorfismos de $\mathbb{S}^{1}$}

Consideremos o círculo $\mathbb{S}^{1}=\mathbb{R} / \mathbb{Z}$, a aplicação de recobrimento $\pi: \mathbb{R} \rightarrow \mathbb{S}^{1}$, um homeomorfismo $f: \mathbb{S}^{1} \rightarrow \mathbb{S}^{1}$, que preserva orientação e $F: \mathbb{R} \rightarrow \mathbb{R}$, um levantamento de $f$, isto é, $\pi \circ F=f \circ \pi$. A seguinte definição foi introduzida por H. Poincaré em [Poi52].

Definição 2.1. Definimos o número de rotação de $f$ como

$$
\rho(f)=\pi(\tau(F))
$$

onde

$$
\tau(F)=\lim _{n \rightarrow \infty} \frac{F^{n}(x)-x}{n}, \quad \text { para cada } x \in \mathbb{R}
$$

Pode-se mostrar que o limite (2.1) existe para cada $x \in \mathbb{R}$, que o número $\rho(f)$ não depende de $x$ e portanto, está bem definido.

Os resultados abaixo, ver [Poi52] e [Den32], explicitam a relação entre o número de rotação e a dinâmica.

Proposição 2.2. Se o número de rotação de $f$ é racional, então $f$ tem pelo menos uma órbita periódica, e todas as órbitas periódicas tem o mesmo período.

Proposição 2.3. Se o número de rotação $\alpha$ de $f$ é irracional, então não existem órbitas periódicas para $f$. Além disso, $f$ é semi-conjugado à rotação $R_{\alpha}$. 
Outro resultado relevante que merece ser citado é a seguinte proposição que afirma que o número de rotação é um invariante topológico da dinâmica.

Proposição 2.4. Sejam $f, h$ homeomorfismos do círculo que preservam orientação. Se $f, h$ são topologicamente conjugados, então $\rho(f)=\rho(h)$.

Porém, o exemplo de Denjoy mostra que existe uma aplicação do círculo com número de rotação irracional que não é conjugada a uma rotação irracional. Denjoy também mostra que homeomorfismos com regularidade $C^{2}$, que preservam orientação com número de rotação irracional, são conjugados a rotações de ângulo irracional. Neste caso temos uma descrição topologicamente completa da dinâmica.

\subsection{Conjunto de rotação em variedades compactas}

Generalizações da definição de número de rotação de Poincaré, seguem entre outros, das ideias em [Sch57]. Por exemplo, M. Pollicot estende esse conceito para homeomorfismos em variedades conexas e compactas, ver [Pol92]. No caso de homeomorfismos do toro homotópicos à identidade, uma definição que se mostrou útil foi dada por M. Misiurewicz e K. Ziemian [MZ89].

Nesta seção, vamos enunciar as definições de intervalo de rotação e conjunto de rotação para homeomorfismos do anel e do toro respectivamente. Antes lembremos os seguintes conceitos:

Definição 2.5. Seja $M$ um espaço topológico. Dizemos que dois homeomorfismos $f, h$ : $M \rightarrow M$ são homotópicos, se existe uma aplicação contínua

$$
H: M \times[0,1] \rightarrow M
$$

chamada homotopia entre $f$ e $h$, tal que $H(x, 0)=f(x)$ e $H(x, 1)=h(x)$, para cada $x \in M$.

Se $H$ é uma homotopia entre $f$ e h, ela define uma família de aplicações contínuas $H_{t}: M \rightarrow M$, dadas por $H_{t}(x)=H(x, t)$.

Dizemos que dois homeomorfismos $f, h: M \rightarrow M$ são isotópicos, se existe uma homotopia $H$ entre $f$ e $h$, tal que para cada $t \in[0,1]$ a aplicação $H_{t}: M \rightarrow M$ é um homeomorfismo. Tal homotopia $H$ é chamada de isotopia.

Um resultado de Epstein [Eps66] mostra que se um homeomorfismo de uma superfície conexa e triangulável é homotópico à identidade, então é isotópico à identidade. 


\section{Homeomorfismos do anel}

Sejam $A=\mathbb{S}^{1} \times[0,1]$ o anel fechado, $\hat{A}=\mathbb{R} \times[0,1]$ o recobrimento universal de $A$, $\operatorname{Pr}_{1}: \hat{A} \rightarrow \mathbb{R}$ a projeção sobre a primeira componente, $f: A \rightarrow A$ um homeomorfismo isotópico à identidade, ou seja, preserva orientação e preserva as componentes da fronteira, e seja $\hat{f}: \hat{A} \rightarrow \hat{A}$ um levantamento de $f$.

Definição 2.6. O conjunto de rotação de $\hat{f}$, denotado por $R(\hat{f})$, se define como o conjunto de pontos $w \in \mathbb{R}$, tais que existem sequências $\left(n_{i}\right)_{i \in \mathbb{N}} \in \mathbb{N} e\left(x_{i}\right)_{i \in \mathbb{N}} \in \hat{A}$, com $n_{i} \rightarrow \infty$ quando $i \rightarrow \infty$, e

$$
\lim _{i \rightarrow \infty} \frac{\operatorname{Pr}_{1}\left(\hat{f}^{n_{i}}\left(x_{i}\right)-x_{i}\right)}{n_{i}}=w
$$

Pode-se mostrar que $R(\hat{f})$ é um segmento de reta compacto, não vazio e é invariante com respeito à conjugação.

\section{Homeomorfismos do toro}

Estamos interessados no estudo dos homeomorfismos do toro. Denotemos este conjunto por hom $\left(\mathbb{T}^{2}\right)$. Inicialmente discutimos a classificação para estes homeomorfismos, segundo a sua classe de homotopia.

\section{Classes de homotopia sobre $\mathbb{T}^{2}$}

Sejam $M_{2}(\mathbb{Z})$ o conjunto das matrizes quadradas inteiras de ordem 2,

$$
\begin{aligned}
G L(2, \mathbb{Z}) & =\left\{A \in M_{2}(\mathbb{Z}) ; \operatorname{det}(A) \neq 0\right\} \quad \mathrm{e} \\
S L(2, \mathbb{Z}) & =\left\{A \in M_{2}(\mathbb{Z}) ; \operatorname{det}(A)=1\right\} .
\end{aligned}
$$

Se $A \in S L(2, \mathbb{Z})$, sabemos que cada matriz $A$ representa uma transformação linear dada por $(\tilde{x}, \tilde{y}) \mapsto A(\stackrel{\tilde{x}}{\tilde{y}})$. Esta transformação linear define um automorfismo linear do toro $f_{A}$ : $\mathbb{T}^{2} \rightarrow \mathbb{T}^{2}$, dado por $f_{A}(x, y)=A(\underset{\tilde{y}}{\tilde{y}})\left(\bmod \mathbb{Z}^{2}\right)$. Vemos que o automorfismo linear do toro $f_{A}$ é um homeomorfismo, onde a transformação linear representada por $A^{-1}$, define o automorfismo linear do toro $f_{A}^{-1} \operatorname{como} f_{A}^{-1}(x, y)=A^{-1}\left(\begin{array}{c}\tilde{x} \\ \tilde{y}\end{array}\right)\left(\bmod \mathbb{Z}^{2}\right)$.

Reciprocamente, um homeomorfismo $f: \mathbb{T}^{2} \rightarrow \mathbb{T}^{2}$, induz uma aplicação no grupo fundamental do toro $f_{*}: \pi_{1}\left(\mathbb{T}^{2}\right) \rightarrow \pi_{1}\left(\mathbb{T}^{2}\right)$, definida por $f_{*}([\alpha])=[f \circ \alpha]$. Como $\pi_{1}\left(\mathbb{T}^{2}\right) \cong \mathbb{Z}^{2}$, podemos identificar $f_{*}$ com um único elemento $A_{f} \in G L(2, \mathbb{Z})$. Se $f$ preserva a orientação, então $A_{f} \in S L(2, \mathbb{Z})$.

Para uma demonstração da seguinte Proposição, podemos ver [BR07]. 
Proposição 2.7. A aplicação $f \mapsto f_{*}$ induz uma bijeção entre as classes de isotopia de homeomorfismos que preservam orientação do toro e $S L(2, \mathbb{Z})$. A inversa desta bijeção é a aplicação que a cada elemento $A \in S L(2, \mathbb{Z})$ associa a classe da isotopia do homeomorfismo $f_{A}$.

A proposição anterior implica em particular:

Corolário 2.8. Cada homeomorfismo $f \in$ hom $\left(\mathbb{T}^{2}\right)$ é isotópico ao único homeomorfismo linear do toro $f_{A}$, onde $A=A_{f}$.

Podemos concluir que a classificação dos homeomorfismos do toro, segundo sua classe de homotopia, está dada pela classificação dos homeomorfismos lineares do toro.

\section{Classificação dos homeomorfismos lineares do toro}

Usando a notação acima, $f_{A}$ é o homeomorfismo linear do toro definido por $A$, que preserva orientação. Vemos que o comportamento dinâmico do homeomorfismo linear $f_{A}$, depende dos autovalores $\lambda_{1}$ e $\lambda_{2}$ da matriz $A$. Assim, temos os seguintes casos:

1. Primeiro, suponhamos que $\left|\lambda_{i}\right|=1$, para $i=1,2$. Denotemos $m g(\lambda)$ a multiplicidade geométrica de $\lambda$. Então, existem duas possibilidades:

(a) Se $A$ tem um único autovalor $\lambda=\lambda_{1}=\lambda_{2}$, com $m g(\lambda)=1$, então ou $\lambda=1$ ou $\lambda=-1$. Neste caso a matriz $A$ é conjugada a uma matriz do tipo $\left(\begin{array}{cc} \pm 1 & m \\ 0 & \pm 1\end{array}\right)$, onde $m \in \mathbb{Z}_{*}$. O homeomorfismo $f_{A}$ é chamado Redutível. Se $\lambda=1$, o homeomorfismo $f_{A}$ é chamado Dehn twist.

(b) Se $A$ tem um único autovalor $\lambda=\lambda_{1}=\lambda_{2}$, com $m g(\lambda)=2$, então ou $\lambda=1 \mathrm{ou}$ $\lambda=-1$. Ou, se $A$ tem autovalores distintos, então são complexos. Em qualquer caso os autovalores são raízes da unidade e existe $n \leq 6$, tal que $f_{A}^{n}=I d$ e $f_{A}$ é de Ordem finita.

2. Agora, suponhamos que $\left|\lambda_{i}\right| \neq 1$, para $i=1$, 2. Então os autovalores $\lambda$ e $\lambda^{-1}$ são reais (irracionais), com $|\lambda|<1$ e $\left|\lambda^{-1}\right|>1$. Neste caso $A$ é chamada hiperbólica e $f_{A}$ Anosov linear.

Resumindo, temos uma classificação para os homeomorfismos do toro em três classes de homotopia, com propriedades distintas.

Teorema 2.9. Se $f \in \operatorname{hom}\left(\mathbb{T}^{2}\right)$, então f é homotótico a um dos seguintes homeomorfismos:

1. Ordem finita. 
2. Redutivel.

3. Anosov Linear.

Observação 2.10. Se $f_{A}$ é Redutível, então $f_{A}^{2}$ é Denh twist.

\section{Conjunto de rotação para homeomorfismos homotópicos à Denh Twist}

Consideremos homeomorfismos homotópicos à Denh twist,

$$
\left(x_{1}, x_{2}\right) \mapsto\left(x_{1}+m x_{2}, x_{2}\right)\left(\bmod \mathbb{Z}^{2}\right),
$$

para algum $m \in \mathbb{Z}_{*}$. Denotemos esse conjunto por $D T\left(\mathbb{T}^{2}\right)$, e por $D T\left(\mathbb{S}^{1} \times \mathbb{R}\right)$ o conjunto dos levantamentos $\widehat{f}$ de elementos de $D T\left(\mathbb{T}^{2}\right)$ ao cilindro vertical, isto é, para cada $\left(z_{1}, z_{2}\right) \in \mathbb{Z}^{2}$,

$$
\widehat{f}\left(\left(\widehat{x}_{1}, \widehat{x}_{2}\right)+\left(z_{1}, z_{2}\right)\right)=\widehat{f}\left(\widehat{x}_{1}, \widehat{x}_{2}\right)+\eta_{m}\left(z_{1}, z_{2}\right),
$$

onde $\eta_{m}\left(z_{1}, z_{2}\right)=\left(z_{1}+m z_{2}, z_{2}\right)$.

Denotemos também a projeção na segunda variável por $p_{2}: \mathbb{S}^{1} \times \mathbb{R} \rightarrow \mathbb{R}, p_{2}\left(\widehat{x_{1}}, \widehat{x_{2}}\right)=\widehat{x_{2}}$. A seguinte definição está dada em [Doe97] e [AZ02].

Definição 2.11. Sejam $f \in D T\left(\mathbb{T}^{2}\right)$ e $\widehat{f} \in D T\left(\mathbb{S}^{1} \times \mathbb{R}\right)$ um levantamento de $f$. Definimos o conjunto de rotação vertical, da seguinte forma:

$$
\rho_{v}(\widehat{f})=\bigcap_{j \geqslant 1} \overline{\bigcup_{i \geqslant j}\left\{\frac{p_{2} \circ \widehat{f}^{i}(\widehat{x})-p_{2}(\widehat{x})}{i} ; x \in \mathbb{S}^{1} \times \mathbb{R}\right\}} .
$$

Este conjunto é um intervalo fechado. Ver [Doe97]. 


\section{Homeomorfismos homotópicos à identidade}

Ao longo deste trabalho, vamos trabalhar com homeomorfismos do toro homotópicos à identidade. Denotamos esse conjunto por $\operatorname{hom}_{0}\left(\mathbb{T}^{2}\right)$.

Consideremos $f \in \operatorname{hom}_{0}\left(\mathbb{T}^{2}\right), \pi: \mathbb{R}^{2} \rightarrow \mathbb{T}^{2}$, a aplicação de recobrimento canônica e $\tilde{f}: \mathbb{R}^{2} \rightarrow \mathbb{R}^{2}$ um levantamento de $f$; isto é, para $x \in \mathbb{R}^{2}$,

$$
\pi \circ \tilde{f}(x)=f \circ \pi(x) .
$$

Então, $\tilde{f}$ é da forma $\tilde{f}=I d+\varphi$, onde $\varphi: \mathbb{R}^{2} \rightarrow \mathbb{R}^{2}$ é biperiódica de período um, assim, se $z \in \mathbb{Z}^{2}, \varphi(x+z)=\varphi(x)$. Logo, $\tilde{f}$ satisfaz a condição:

$$
\tilde{f}(x+z)=\tilde{f}(x)+z, \forall z \in \mathbb{Z}^{2} \text { e } \forall x \in \mathbb{R}^{2} .
$$

Observemos que se $\tilde{f}_{1}$ e $\tilde{f}_{2}$ são levantamentos de $f$, então existe $v \in \mathbb{Z}^{2}$, tal que

$$
\tilde{f}_{1}(x)=\tilde{f}_{2}(x)+v
$$

\subsection{Conjunto de rotação no toro}

As seguintes definições foram introduzidas por M. Misiurewics e K. Ziemian em [MZ89].

Definição 2.12. Seja $x \in \mathbb{T}^{2}$. $O$ vetor de rotação de $x$ por $\tilde{f}$ é definido como

$$
\lim _{n \rightarrow \infty} \frac{\tilde{f}^{n}(\tilde{x})-\tilde{x}}{n}
$$

quando este limite existir para algum $\tilde{x} \in \pi^{-1}(x)$. Neste caso, o limite (2.3) é denotado por $\rho(\tilde{f}, x)$.

Observemos que esta definição não depende de $\tilde{x}$, dado que $\tilde{f}$ satisfaz a igualdade (2.2).

Definição 2.13. Definimos o conjunto de rotação pontual de $\tilde{f}$ como

$$
\rho_{p}(\tilde{f})=\bigcup_{x \in \mathbb{T}^{2}} \rho(\tilde{f}, x)
$$

Definição 2.14. O conjunto de rotação de $\tilde{f}$, denotado por $\rho(\tilde{f})$, se define como o conjunto de pontos $v \in \mathbb{R}^{2}$, tais que existem sequências $\left(n_{i}\right)_{i \in \mathbb{N}} \in \mathbb{N}$ e $\left(x_{i}\right)_{i \in \mathbb{N}} \in \mathbb{R}^{2}$, com $n_{i} \rightarrow \infty$ quando $i \rightarrow \infty$, e

$$
\lim _{i \rightarrow \infty} \frac{\tilde{f}^{n_{i}}\left(x_{i}\right)-x_{i}}{n_{i}}=v
$$


Este conjunto é equivalente ao conjunto:

$$
\bigcap_{j \geqslant 1} \overline{\bigcup_{i \geqslant j}\left\{\frac{\tilde{f}^{i}(x)-x}{i} ; x \in \mathbb{R}^{2}\right\}} .
$$

Propriedade 2.15. Para cada $\tilde{f}$, existe $M>0$, tal que

$$
\left\|\frac{\tilde{f}^{n_{i}}\left(x_{i}\right)-x_{i}}{n_{i}}\right\| \leqslant M, \quad \forall n_{i} \in \mathbb{N}, \quad \text { e } \forall x_{i} \in \mathbb{R}^{2} .
$$

Demonstração. Como $\varphi=\tilde{f}-I d$ é biperiódica, existe $M>0$ tal que,

$$
\left\|\tilde{f}\left(x_{i}\right)-x_{i}\right\| \leqslant M, \quad \forall x_{i} \in \mathbb{R}^{2} .
$$

Por outro lado, para cada $n_{i} \in \mathbb{N}$,

$$
\frac{\tilde{f}^{n_{i}}\left(x_{i}\right)-x_{i}}{n_{i}}=\frac{1}{n_{i}} \sum_{i=0}^{n_{i}-1} \tilde{f}\left(\tilde{f}^{i}\left(x_{i}\right)\right)-\tilde{f}^{i}\left(x_{i}\right) .
$$

Logo,

$$
\begin{aligned}
\left\|\frac{\tilde{f}^{n_{i}}\left(x_{i}\right)-x_{i}}{n_{i}}\right\| & \leqslant \frac{1}{n_{i}} \sum_{i=0}^{n_{i}-1}\left\|\tilde{f}\left(\tilde{f}^{i}\left(x_{i}\right)\right)-\tilde{f}^{i}\left(x_{i}\right)\right\| \\
& \leqslant \frac{1}{n_{i}} \sum_{i=0}^{n_{i}-1} M=M .
\end{aligned}
$$

Observação 2.16. Essa propriedade implica que o conjunto de rotação é limitado. Sendo interseção enumerável de compactos encaixantes, ele é não vazio.

Seja $\phi: \mathbb{T}^{2} \rightarrow \mathbb{R}^{2}$ a função deslocamento definida por $\phi(x)=\tilde{f}(\tilde{x})-\tilde{x}$, para cada $x \in \mathbb{T}^{2}$ e $\tilde{x} \in \pi^{-1}(x)$. Observemos que $\phi(x)=\varphi(\tilde{x})$, para cada $\tilde{x} \in \pi^{-1}(x)$.

Denotemos por $\mathcal{M}(f)$ o conjunto das medidas de probabilidade, borelianas, $f$-invariantes em $\mathbb{T}^{2}$, e por $\mathcal{M}_{e}(f)$ o subconjunto de $\mathcal{M}(f)$ das medidas ergódicas.

Definição 2.17. Seja $\mu \in \mathcal{M}(f)$. O vetor de rotação de $\mu$ é definido como

$$
\rho(\mu, \tilde{f})=\int_{\mathbb{T}^{2}} \phi d \mu
$$


Definimos também os conjuntos:

$$
\begin{aligned}
\rho_{m}(\tilde{f}) & =\{\rho(\mu, \tilde{f}) ; \mu \in \mathcal{M}(f)\} e \\
\rho_{e}(\tilde{f}) & =\left\{\rho(\mu, \tilde{f}) ; \mu \in \mathcal{M}_{e}(f)\right\} .
\end{aligned}
$$

Lembremos a seguinte definição, que será usada ao longo deste capítulo.

Definição 2.18. Um ponto $x$ de um conjunto convexo $C$ é chamado ponto extremal, se $x$ não é um ponto interior de nenhum segmento de reta contido em $C$, isto é, se não existem pontos $y, z \in C$ e $\lambda \in(0,1)$ tais que $x=\lambda y+(1-\lambda) z$, exceto quando $x=y=z$.

Denotemos por $\operatorname{Conv}(A)$ o fecho convexo de um conjunto $A$, e $\operatorname{Ext}(A)$ o conjunto dos pontos extremais de $A$. O seguinte resultado encontra-se por exemplo em [Roc70].

Corolário 2.19. Um conjunto convexo, limitado e fechado é o fecho convexo dos seus pontos extremais.

Proposição 2.20 ([MZ89]). Se $\mu \in \mathcal{M}_{e}(f)$ e $v=\rho(\mu, \tilde{f})$, então $v=\rho(\tilde{f}, x)$, para $\mu$-q.t. $x \in \mathbb{T}^{2}$.

Demonstração. Como $\phi$ é contínua, $\phi \in \mathcal{L}_{1}(\mu)$, então pelo Teorema Ergódico de Birkoff, para $\mu$-q.t. $x \in T^{2}$ e cada $\tilde{x} \in \pi^{-1}(x)$,

$$
v=\int_{\mathbb{T}^{2}} \phi d \mu=\lim _{n \rightarrow \infty} \frac{1}{n} \sum_{k=0}^{n-1} \phi \circ f^{k}(x)=\lim _{n \rightarrow \infty} \frac{\tilde{f}^{n}(\tilde{x})-\tilde{x}}{n} .
$$

Portanto, para $\mu$-q.t. $x \in \mathbb{T}^{2}, v=\rho(\tilde{f}, x)$.

Dizemos que um ponto $v \in \rho(\tilde{f})$ é realizado por um subconjunto $K \subset \mathbb{T}^{2}$, se $K$ for compacto, $f$-invariante e o vetor de rotação $\rho(\tilde{f}, x)=v$, para todo $x \in K$. Em particular, dizemos que um ponto $v \in \rho(\tilde{f})$ é realizado por uma órbita periódica, se $v \in \mathbb{Q}^{2}$ e existem $k \in \mathbb{N}$ e $x \in \mathbb{T}^{2}$, tais que $f^{k}(x)=x$ e $\tilde{f}^{k}(\tilde{x})=\tilde{x}+v$, para $\tilde{x} \in \pi^{-1}(x)$.

\subsection{Propriedades do conjunto de rotação}

Nesta seção vamos enunciar alguns resultados conhecidos no estudo do conjunto de rotação. Em [MZ89], foi mostrado o seguinte:

Teorema 2.21.

$$
\rho(\tilde{f})=\operatorname{Conv}\left(\rho_{p}(\tilde{f})\right)=\operatorname{Conv}\left(\rho_{e}(\tilde{f})\right)=\rho_{m}(\tilde{f}) .
$$


Observação 2.22. Em particular, o conjunto de rotação é compacto (pois é fechado por sua definição) e convexo.

A seguinte proposição mostra propriedades análogas às propriedades do número de rotação de homeomorfismos do círculo.

Proposição 2.23. Se $p \in \mathbb{Z}^{2}$ e $q \in \mathbb{N}$ então,

1. $\rho\left(\tilde{f}^{q}-p\right)=q \rho(\tilde{f})-p$.

2. $\rho\left(\tilde{f}^{q}-p, z\right)=q \rho(\tilde{f}, z)-p$, para cada $z \in \mathbb{R}^{2}$.

3. $\rho_{p}\left(\tilde{f}^{q}-p\right)=q \rho_{p}(\tilde{f})-p$.

4. $\rho_{e}\left(\tilde{f}^{q}-p\right)=q \rho_{e}(\tilde{f})-p$.

5. $\rho\left(\tilde{f}^{-1}\right)=-\rho(\tilde{f})$.

6. $\rho_{e}\left(\tilde{f}^{-1}\right)=-\rho_{e}(\tilde{f})$.

A demonstração do seguinte lema, pode ser encontrado em [KK08].

Lema 2.24. Sejam $f \in \operatorname{hom}_{0}\left(\mathbb{T}^{2}\right), A \in S L(2, \mathbb{Z}), h \in \operatorname{hom}\left(\mathbb{T}^{2}\right)$, isotópico à $f_{A}$, e $\tilde{f}, \tilde{h}$ respectivos levantamentos de $f$ e h. Então,

$$
\rho\left(\tilde{h} \tilde{f} \tilde{h}^{-1}\right)=A \rho(\tilde{f})
$$

Em particular, $\rho\left(\tilde{A} \tilde{f} \tilde{A}^{-1}\right)=A \rho(\tilde{f})$.

F. Beguin formula a seguinte pergunta em suas notas de aula [Beg07]: existe um compacto convexo $K \subset \mathbb{R}^{2}$ que não é o conjunto de rotação de nenhum homeomorfismo homotópico à identidade de $\mathbb{T}^{2}$ ?

Uma questão mais geral é: quais conjuntos compactos e convexos de $\mathbb{R}^{2}$ podem ser o conjunto de rotação de um homeomorfismo homotópico à identidade de $\mathbb{T}^{2}$ ?

Sabemos que qualquer ponto pode ser um vetor de rotação, como veremos na subseção de Pseudo-rotações.

Entre outros resultados em relação a que conjuntos podem ser conjuntos de rotação, temos resposta no trabalho de J. Franks e M. Misiurewicz [FM90], no caso de fluxos do toro $\mathbb{T}^{2}$, onde o interior do conjunto de rotação é necessariamente vazio. Neste caso, eles mostram que o conjunto de rotação tem que ser uma das seguintes possibilidades: ou um único ponto, ou um segmento de reta com inclinação racional e infinitos pontos racionais, ou um segmento de reta com inclinação irracional e um ponto extremal racional. Eles também exibem exemplos de conjuntos de rotação, para todos estes casos. 
Também encontramos os trabalhos de Kwapisz sobre polígonos como conjuntos de rotação:

Proposição 2.25 ([Kwa92]). Qualquer polígono convexo com vértices racionais, pode ser realizado como um conjunto de rotação.

E ainda, em [Kwa95], mostra-se um exemplo onde o conjunto de rotação tem uma quantidade enumerável infinita de vértices, $\operatorname{logo} \rho(\tilde{f})$ não pode ser um polígono.

Recentemente P. Boyland, A. De Carvalho e T. Hall, construíram uma família de homeomorfismos do toro, homotópicos à identidade, cujos conjuntos de rotação podem ser descritos explicitamente. Ver [BCH14]. Nesta família todos os conjuntos de rotação são do tipo descrito ou em [Kwa92] ou em [Kwa95].

Franks e Misiurewicz conjecturaram que um segmento de reta $L$ não pode ser realizado como um conjunto de rotação de um homeomorfismo do toro nas seguintes condições:

i. O segmento de reta $L$ tem inclinação irracional e um ponto racional no seu interior relativo.

ii. O segmento de reta $L$ tem inclinação racional, sem pontos racionais.

iii. O segmento de reta $L$ tem inclinação irracional sem pontos racionais.

O teorema seguinte ([CT15], Teorema D), implica a conjectura para o caso i.

Teorema 2.26. Sejam $f \in \operatorname{hom}_{0}\left(\mathbb{T}^{2}\right)$ e $\tilde{f}$ um levantamento de $f$. O conjunto $\rho(\tilde{f})$ não pode conter um segmento de reta com inclinação irracional que contenha um ponto racional no seu interior relativo.

A. Avila anunciou um contraexemplo para o caso iii.

\section{Implicações para a dinâmica}

Vejamos que algumas propriedades geométricas de $\rho(\tilde{f})$ implicam resultados sobre a dinâmica de $f$. Uma questão que tem sido bastante estudada, além das apresentadas na introdução, em relação à informação dinâmica que podemos obter, conhecendo o conjunto de rotação é:

Questão 3. Quais pontos no conjunto de rotação podem ser realizados?

M. Misiurevicz e K. Ziemian [MZ89], respondem essa pergunta para pontos extremais do conjunto de rotação: 
Proposição 2.27. Todo ponto extremal de $\rho(\tilde{f})$ é realizado.

Além disso, eles mostram que pontos extremais racionais são realizados por órbitas periódicas.

Teorema 2.28 ([MZ89]). Se $\left(\frac{p_{1}}{q}, \frac{p_{2}}{q}\right) \in \rho_{e}(\tilde{f}) \cap \mathbb{Q}^{2}$ e m.c.d. $\left(p_{1}, p_{2}, q\right)=1$, então existe um ponto periódico $x \in \mathbb{T}^{2}$ para $f$ de período $q$, tal que

$$
\tilde{f}^{q}(y)=y+\left(p_{1}, p_{2}\right)
$$

para cada $y \in \pi^{-1}(x)$.

Mais exatamente o teorema anterior é uma consequência do seguinte teorema de J. Franks. Denotemos por Fix $(h)$ o conjunto dos pontos fixos de $h$.

Teorema 2.29 ([Fra88]). Se $0 \in \rho_{e}(\tilde{f})$, então Fix $(\tilde{f}) \neq \emptyset$.

Em breve veremos outros resultados conhecidos que respondem parcialmente à Questão 3 .

Dado que o conjunto de rotação é um subconjunto compacto e convexo de $\mathbb{R}^{2}$, ele pode ser dos seguintes tipos:

1. Um único ponto.

2. Um segmento de reta.

3. Ter interior não vazio.

Abaixo discutimos propriedades particulares a cada um destes casos.

Denotemos por $\langle$,$\rangle o produto interno canônico em \mathbb{R}^{2}$ e $\operatorname{Pr}_{v}: \mathbb{R}^{2} \rightarrow \mathbb{R}$ a projeção ortogonal dada por:

$$
\operatorname{Pr}_{v}(x)=\frac{\langle x, v\rangle}{\|v\|} .
$$

Se $v=(a, b) \in \mathbb{R}^{2}$, denotamos o vetor ortogonal $v^{\perp}=(-b, a)$.

\section{Pseudo-rotações}

Definição 2.30. Dizemos que $f$ é uma pseudo-rotação se $\rho(\tilde{f})=\{v\}$, ou seja o conjunto de rotação contém um único ponto. Se $v \in \mathbb{Q}^{2}$, dizemos que é uma pseudo-rotação racional, se $v \in \mathbb{R}^{2} \backslash \mathbb{Q}^{2}$, dizemos que é uma pseudo-rotação irracional. Por fim, dizemos que $f$ é irrotacional se $\rho(\tilde{f})=0$. 
Os exemplos mais simples de pseudo-rotação são as translações:

Exemplo 2.31. Sejam $f \in \operatorname{hom}_{0}\left(\mathbb{T}^{2}\right)$ e $\tilde{f}: \mathbb{R}^{2} \rightarrow \mathbb{R}^{2}$ um levantamento de $f$ definido por:

$$
\tilde{f}(x)=x+v
$$

para algum $v \in \mathbb{R}^{2}$. Então,

$$
\rho(\tilde{f})=\{v\}
$$

Logo, qualquer ponto em $\mathbb{R}^{2}$ pode ser o vetor de rotação de uma translação, como comentamos anteriormente.

Entre outros estudos sobre a dinâmica de pseudo-rotações, podemos citar alguns trabalhos de A. Koropecki e F. Tal. Em [KT12] é apresentado um exemplo de um difeomorfismo ergódico e irrotacional que preserva área com órbitas ilimitadas em todas as direções.

No próximo teorema, os autores classificam as pseudo-rotações racionais com medida invariante de suporte total. Antes de enunciar o teorema, precisamos introduzir os seguintes conceitos:

Um conjunto é totalmente essencial, se contém o complemento de alguma união disjunta de discos topológicos.

Dado $f \in \operatorname{hom}\left(\mathbb{T}^{2}\right)$, dizemos que $f$ é Hamiltoniano, se preserva uma medida $\mu$ de suporte total e tem um levantamento, tal que o vetor de rotação de $\mu$ é nulo.

Teorema 2.32 ([KT14a]). Seja $f: \mathbb{T}^{2} \rightarrow \mathbb{T}^{2}$ um homeomorfismo Hamiltoniano e seja $\tilde{f}$ seu levantamento irrotacional. Então, uma das seguintes afirmações é válida:

1. O conjunto Fix $(f)$ é totalmente essencial.

2. Cada ponto de $\mathbb{R}^{2}$ tem uma $\tilde{f}$-órbita limitada.

3. $\tilde{f}$ tem deslocamento uniformemente limitado numa direção racional, isto é, existem $v \in \mathbb{Z}_{*}^{2}$ e $M>0$ tais que

$$
\left|\left\langle\tilde{f}^{n}(z)-z, v\right\rangle\right| \leqslant M
$$

para cada $z \in \mathbb{R}^{2}$ e $n \in \mathbb{Z}$.

F. Tal apresenta uma melhora do teorema anterior, mediante o seguinte resultado:

Teorema 2.33 ([Tal14]). Sejam $f: \mathbb{T}^{2} \rightarrow \mathbb{T}^{2}$ um homeomorfismo Hamiltoniano e $\tilde{f}$ seu levantamento irrotacional. Se Fix $(f)$ está contido num disco topológico, então existe $v \in \mathbb{Z}^{2}$ tal que o diâmetro de cada órbita por $\tilde{f}$ é uniformemente limitado, na direção $v$. 
Recentemente, P. Le Calvez e F. Tal mostraram que, nas mesmas hipóteses do Teorema 2.33, o diâmetro de cada órbita por $\tilde{f}$ é uniformemente limitado. Ver ([CT15], Corolário H).

No estudo de pseudo-rotações, aparece uma outra questão natural. Assim como no caso de homeomorfismos do círculo, queremos saber quando $f$, uma pseudo-rotação com vetor de rotação $v$, é conjugada a uma rotação $R_{v}$. Nesta direção os trabalhos de T. Jäger são uma boa referência: em [Jag09a] é dado um teorema de classificação para pseudo-rotações conservativas, análoga à classificação dada por H. Poincaré; e em [Jag09b] é mostrado que pseudo-rotações irracionais, não errantes, são transitivas.

Para outros trabalhos sobre pseudo-rotações irracionais, podemos citar [BCJR09] e a proposição seguinte:

Proposição 2.34 ([BCRP04]). Seja $f$ uma pseudo-rotação. Então $R_{v}$ pertence ao fecho da classe de conjugação de $f$.

\section{Segmentos de rotação}

Consideramos o caso em que $\rho(\tilde{f})$ é um segmento de reta.

Exemplo 2.35. Sejam $\varphi: \mathbb{R} \rightarrow[a, b]$ uma função contínua, de período um, definida por:

$$
\varphi(s)= \begin{cases}\left(\frac{b-a}{4}\right) s(1-s)+a, & \text { se } 0 \leqslant s \leqslant 1 \\ \varphi(s \bmod 1), & \text { se c.c. }\end{cases}
$$

$E$

$$
\tilde{f}(x, y)=(x+\varphi(y), y) .
$$

Como $\tilde{f}^{n}(x, y)-(x, y)=n(\varphi(y), 0)$ então,

$$
\rho(\tilde{f})=[a, b] \times\{0\} .
$$

Podemos citar os seguintes trabalhos, em relação à Questão 3.

Teorema 2.36 ([Fra95]). Seja $\tilde{f}$ um levantamento de $f$ que preserva área. Se $\rho(\tilde{f})$ é um segmento de reta e $v \in \rho(\tilde{f})$ é um ponto racional, então existe $x \in \mathbb{R}^{2}$ tal que $\pi(x) \in \mathbb{T}^{2}$ é um ponto periódico para $f e$

$$
v=\lim _{n \rightarrow \infty} \frac{\tilde{f}^{n}(x)-x}{n} .
$$

Proposição 2.37 ([JZ98]). Seja $\rho(\tilde{f})$ um segmento de reta com inclinação irracional. Se $v \in \rho(\tilde{f})$ é um ponto de coordenadas racionais, então existe um ponto periódico $x \in \mathbb{T}^{2}$, tal que $v$ é realizado pela órbita de $x$. 
Uma definição que aparece nos estudos deste tipo de conjunto de rotação é a seguinte:

Definição 2.38. Dizemos que $f \in \operatorname{hom}_{0}\left(\mathbb{T}^{2}\right)$ é anular, se existe algum levantamento $\tilde{f}$ de $f$ tal que o deslocamento de $\tilde{f}$ em alguma direção $v \in \mathbb{Z}_{*}^{2}$ é uniformemente limitado, isto é, se existe $M>0$ tal que

$$
\left|\left\langle\tilde{f}^{n}(x)-x, v\right\rangle\right| \leqslant M,
$$

para cada $x \in \mathbb{R}^{2}$ e $n \in \mathbb{Z}$.

Com esta definição também encontramos a seguinte pergunta:

Questão 4. Se $\rho(\tilde{f})$ é um segmento de reta, com pontos racionais, então $f$ é anular?

Esta pergunta foi respondida afirmativamente, primeiro no contexto conservativo:

Teorema 2.39 ([GKT14]). Seja $\tilde{f}$ um levantamento de um homeomorfismo $f$ que preserva área com $\rho(\tilde{f})=\{0\} \times[a, b]$, onde $a<b$. Então $f$ é anular na direção $v=(0,1)$.

E depois no caso geral:

Teorema 2.40 ([Dav13]). Se $\rho(\tilde{f})$ é um segmento de reta com inclinação racional, com pontos racionais, então $f^{k}$ é anular, para algum $k \in \mathbb{N}$.

O teorema anterior será usado nas consequências do nosso resultado principal, no Capítulo 4 .

\section{Interior não vazio}

Agora vamos considerar $\operatorname{int}(\rho(\tilde{f})) \neq \emptyset$.

Exemplo 2.41. Sejam $\varphi: \mathbb{R} \rightarrow[0,1]$ uma função contínua, de período um, definida por:

$$
\varphi(s)= \begin{cases}2 s, & \text { se } 0 \leqslant s \leqslant \frac{1}{2} \\ 2-2 s, & \text { se } \frac{1}{2} \leqslant s \leqslant 1 \\ \varphi(s \bmod 1), & \text { se c.c. }\end{cases}
$$

$E$

$$
\tilde{f}(x, y)=(x+\varphi(y), y+\varphi(x)) .
$$

Vemos que se $s \in\left\{0, \frac{1}{2}\right\}, \varphi(s) \in \mathbb{Z}$, então $\tilde{f}^{n}(x, y)-(x, y)=n(\varphi(y), \varphi(x))$, para $(x, y) \in$ $\left\{0, \frac{1}{2}\right\}^{2}$. 
Logo, para $x, y \in\left\{0, \frac{1}{2}\right\}$ temos,

$$
\{(\varphi(y), \varphi(x))\}=\rho(\tilde{f},(x, y)) \subset \rho(\tilde{f}) .
$$

Portanto, $\{(0,0),(0,1),(1,0),(1,1)\} \subset \rho(\tilde{f})$. Como $\rho(\tilde{f})$ é convexo, temos que $[0,1]^{2} \subset \rho(\tilde{f})$. Por outro lado, como $(\tilde{f}-I d)$ é biperiódica de período um, vemos que para cada $(x, y) \in \mathbb{R}^{2}$ e para $i=1,2$,

$$
0 \leqslant \operatorname{Pr}_{i}[\tilde{f}(x, y)-(x, y)] \leqslant 1 .
$$

Logo, $0 \leqslant \operatorname{Pr}_{i}\left[\tilde{f}^{n}(x, y)-(x, y)\right] \leqslant n$, e portanto, $\operatorname{Pr}_{i}(\rho(\tilde{f})) \subset[0,1]$, para $i=1,2$.

Assim,

$$
\rho(\tilde{f})=[0,1]^{2}
$$

Com respeito à Questão 3, para este caso, conhecemos os seguintes resultados:

Teorema 2.42 ([Fra89]). Se $v \in \operatorname{int}(\rho(\tilde{f}))$ é um vetor com coordenadas racionais, então existe um ponto $x \in \mathbb{R}^{2}$, tal que $\pi(x) \in \mathbb{T}^{2}$ é um ponto periódico para $f e$

$$
v=\lim _{n \rightarrow \infty} \frac{\tilde{f}^{n}(x)-x}{n} .
$$

M. Misiurewicz e K. Ziemian, fornecem uma resposta mais geral, para qualquer ponto no interior do conjunto de rotação:

Teorema 2.43 ([MZ91]). Se $v \in \operatorname{int}(\rho(\tilde{f}))$ então,

i. Existe um subconjunto $X \subset \mathbb{T}^{2}, f$-invariante, fechado, não vazio, tal que $\rho(\tilde{f}, y)=v$, para $y \in X$.

ii. Existe uma medida $\mu \in M_{e}(f)$, com $\int \phi d \mu=v$.

Outro resultado bem conhecido em relação à dinâmica de $f$ é o seguinte:

Teorema 2.44 ([LM91]). Se $\tilde{f}$ tem conjunto de rotação com interior não vazio, então $f$ tem entropia topológica positiva.

No contexto de homeomorfismos não errantes, temos o Teorema C em [KT14b]. Antes enunciamos as seguintes definições:

Definição 2.45. Definimos a taxa de difusão de $\tilde{f}$ num disco aberto $U$, como o raio interno do fecho convexo de $\rho(\tilde{f}, U)$. E definimos os seguintes conjuntos: $C(f)$ o conjunto de todos os $x \in \mathbb{T}^{2}$ tais que cada vizinhança de $x$ tem taxa de difusão positiva e $E(f)=\mathbb{T}^{2} \backslash C(f)$.

Um conjunto de $\mathbb{T}^{2}$ é essencial, se não está contido num disco topológico aberto. Um ponto $x \in \mathbb{T}^{2}$ é essencial por $f$, se a órbita de cada vizinhança de $x$ por $f$ é um subconjunto 
essencial de $\mathbb{T}^{2}$. O conjunto dos pontos essenciais por $f$ é denotado Ess $(f)$ e o conjunto dos pontos inessenciais é Ine $(f)=\mathbb{T}^{2} \backslash \operatorname{Ess}(f)$.

Teorema 2.46. Sejam $f \in \operatorname{hom}_{0}\left(\mathbb{T}^{2}\right)$ não errante e $\tilde{f}$ um levantamento de $f$. Suponha que $\rho(\tilde{f})$ tem interior não vazio. Então,

1. O conjunto $C(f)=E s s(f)$ e $E(f)=\operatorname{Ine}(f)$.

2. O conjunto Ess $(f)$ é externamente transitivo e sensitivo às condições iniciais.

3. Para cada $x \in \operatorname{Ess}(f)$ e qualquer vizinhança $U$ de $x, \operatorname{Conv}(\rho(\tilde{f}, U))=\rho(\tilde{f})$.

Citamos um outro resultado relevante para homeomorfismos cujo conjunto de rotação tem interior não vazio, no qual mostra que as órbitas no levantamento tem seu deslocamento constrangido pelo conjunto de rotação.

Teorema 2.47. Se $\rho(\tilde{f})$ tem interior não vazio, existe $M>0$ tal que, para cada $x \in \mathbb{R}^{2} e$ cada $n \in \mathbb{N}$,

$$
d\left(\tilde{f}^{n}(x)-x, n \rho(\tilde{f})\right)<M
$$

Este resultado, obtido para difeomorfismos $C^{1+\epsilon}$ em [AZ15] e estendido para homeomorfismos em [CT15], é o resultado de deslocamento análogo aos Teoremas 2.33 e 2.40. Um resultado similar também existe para homeomorfismos homotópicos à Dehn twist, ver [AZTG14].

\section{Transitividade no levantamento}

Agora vamos fazer uma breve discussão sobre transitividade para levantamentos de homeomorfismos em superfícies.

Em [Boy09] encontramos um trabalho sobre a dinâmica desses homeomorfismos. F. Tal nos presenta no próximo teorema, uma relação entre um levantamento de um homeomorfismo do toro, homotópico à identidade e transitivo, e seu conjunto de rotação.

Teorema 2.48 ([Tal12]). Se $\tilde{f}$ é transitivo, então $(0,0) \in \operatorname{int}(\rho(\tilde{f}))$.

Podemos ver que se $\tilde{f}$ é um levantamento transitivo de um homeomorfismo $f$ do toro, então $f$ é transitivo. A recíproca nem sempre é verdade. O seguinte resultado permite, em particular, decidir quando um homeomorfismo transitivo do toro é transitivo no seu levantamento.

Teorema 2.49 ([GKT12]). Seja $f$ transitivo. Um levantamento $\tilde{f}$ é transitivo se, e somente se, o conjunto de rotação de $\tilde{f}$ contém à origem no seu interior. 


\subsection{Homeomorfismo anular}

Nesta seção seguimos as ideias em [Ben13].

Definimos o deslocamento de $\tilde{f}$ por,

$$
\operatorname{Desl}(\tilde{f})=\sup _{\substack{n \in \mathbb{Z} \\ x \in \mathbb{R}^{2}}}\left\|\tilde{f}^{n}(x)-x\right\|
$$

Se $v \in \mathbb{R}_{*}^{2}$, definimos o deslocamento de $\tilde{f}$ na direção de $v$ por,

$$
\operatorname{Desl}_{v}(\tilde{f})=\sup _{\substack{n \in \mathbb{Z} \\ x \in \mathbb{R}^{2}}}\left|\operatorname{Pr}_{v}\left(\tilde{f}^{n}(x)-x\right)\right|
$$

Notemos que o deslocamento de $\tilde{f}$ pode ser infinito.

Definição 2.50. Seja $v \in \mathbb{Z}_{*}^{2}$. Dizemos que $\tilde{f}$ é $v$-anular, se o deslocamento de $\tilde{f}$ na direção $v^{\perp}$ é limitado.

Um homeomorfismo $f \in \mathbb{T}^{2}$ é $v$-anular, se existe um levantamento $\tilde{f}$ de $f$ tal que $\tilde{f}$ é $v$-anular. E dizemos que $f$ é anular, se existe $v \in \mathbb{Z}_{*}^{2}$ tal que $f$ é $v$-anular.

Definimos o desvio de $\tilde{f}$ por,

$$
\operatorname{Desv}(\tilde{f})=\sup _{\substack{n \in \mathbb{Z} \\ x, y \in \mathbb{R}^{2}}}\left\|\left(\tilde{f}^{n}(x)-x\right)-\left(\tilde{f}^{n}(y)-y\right)\right\|
$$

Se $v \in \mathbb{R}_{*}^{2}$, definimos o desvio de $\tilde{f}$ na direção de $v$ por,

$$
\operatorname{Desv}_{v}(\tilde{f})=\sup _{\substack{n \in \mathbb{Z} \\ x, y \in \mathbb{R}^{2}}}\left|\operatorname{Pr}_{v}\left[\left(\tilde{f}^{n}(x)-x\right)-\left(\tilde{f}^{n}(y)-y\right)\right]\right| .
$$

Notemos que o desvio de $\tilde{f}$ pode ser infinito.

Observação 2.51. Se $\tilde{f}$ é v-anular, para algum $v \in \mathbb{Z}_{*}^{2}$, então existe algum homeomorfismo conjugado à $\tilde{f}$ que é $(0,1)$-anular. Com efeito, dado $v=(q, p) \in \mathbb{Z}^{2}$, existem inteiros $a, b$, tais que $p a+q b=1$. Consideremos a matriz

$$
A=\left[\begin{array}{cc}
p & -q \\
b & a
\end{array}\right]
$$

Logo, $\operatorname{det}(A)=1$ e como $A \cdot(q, p)=(0,1)$, do Lema 2.24 , concluímos que $A \tilde{f} A^{-1}$ é $(0,1)$ anular. 
Propriedade 2.52. Se $\operatorname{Desv}_{u}(\tilde{f})$ é limitado, para algum $u \in \mathbb{R}_{*}^{2}$, então existe $a \in \mathbb{R}$ tal que, $\operatorname{Pr}_{u}(\rho(\tilde{f}))=\{a\}$.

Neste caso, $\rho(\tilde{f}) \subset L\left(u^{\perp}, a\right):=\left\{t u^{\perp}+a u ; t \in \mathbb{R}\right\}$.

Demonstração. Da definição temos que existe $M>0$, tal que para todo $x, y \in \mathbb{R}^{2}$ e $n \in \mathbb{Z}_{*}$,

$$
\left|\operatorname{Pr}_{u}\left[\left(\tilde{f}^{n}(x)-x\right)-\left(\tilde{f}^{n}(y)-y\right)\right]\right| \leqslant M .
$$

Seja $p=\rho\left(\tilde{f}, x_{0}\right)$, para algum $x_{0} \in \mathbb{T}^{2}, \operatorname{com} \operatorname{Pr}_{u}(p)=a \in \mathbb{R}$. Segue da desigualdade (2.5), que $\lim _{n \rightarrow \infty} \operatorname{Pr}_{u} \frac{\tilde{f}^{n}(y)-y}{n}=a$, para cada $y \in \mathbb{R}^{2}$. Portanto $\{a\}=\operatorname{Pr}_{u}\left(\rho_{p}(\tilde{f})\right)=\operatorname{Pr}_{u}(\rho(\tilde{f})) . \diamond$

Observação 2.53. Se $\tilde{f}$ é v-anular, para algum $v \in \mathbb{Z}_{*}^{2}$, podemos ver que $\operatorname{Desv}_{v^{\perp}}(\tilde{f})$ é limitado e portanto $\rho(\tilde{f}) \subset L(v, a)=\left\{t v+a v^{\perp} ; t \in \mathbb{R}\right\}$.

Proposição 2.54. Se $\rho(\tilde{h})$ é um segmento não degenerado, contido na reta

$$
L(w, b)=\left\{t w+b w^{\perp} ; t \in \mathbb{R}\right\},
$$

onde $b=\operatorname{Pr}_{w^{\perp}}(\rho(\tilde{h}))$ e $w \in \mathbb{R}^{2}$, então $\operatorname{Desv}_{v^{\perp}}(\tilde{h})$ é ilimitado, para qualquer $v \in \mathbb{R}^{2}$ não paralelo à $w$.

Demonstração. Suponhamos por contradição que existe $M>0$ tal que, $\operatorname{Desv}_{v^{\perp}}(\tilde{h}) \leqslant M$, então da Propriedade 2.52, sabemos que $\rho(\tilde{h})$ é um subconjunto da reta

$$
L(v, a)=\left\{t v+a v^{\perp} ; t \in \mathbb{R}\right\}
$$

onde $a=\operatorname{Pr}_{v^{\perp}}(p)$, para qualquer $p \in \rho(\tilde{h})$. Então $\rho(\tilde{h}) \subset[L(v, a) \cap L(w, b)]$. Como $v$ e $w$ não são paralelos, concluímos que $\rho(\tilde{h})=\{p\}$. Absurdo!

Proposição 2.55. Se $\tilde{f}$ não é v-anular e $0 \in \operatorname{Pr}_{v^{\perp}}(\rho(\tilde{f}))$, então $\operatorname{Desv}_{v^{\perp}}(\tilde{f})$ é ilimitado.

Demonstração. Suponhamos por contradição que existe $M_{0}>0$, tal que $\operatorname{Desv}_{v^{\perp}}(\tilde{f}) \leqslant M_{0}$. Então, para cada $x, y \in \mathbb{R}^{2}$,

$$
-M_{0} \leqslant \operatorname{Pr}_{v}\left[\left(\tilde{f}^{n}(x)-x\right)-\left(\tilde{f}^{n}(y)-y\right)\right] \leqslant M_{0} .
$$

Como $\tilde{f}$ não é $v$-annular, existem $x_{0} \in \mathbb{R}^{2}$ e $q \in \mathbb{Z}_{*}$, tais que $\left|\operatorname{Pr}_{v^{\perp}}\left(\tilde{f}^{q}\left(x_{0}\right)-x_{0}\right)\right|>2 M_{0}$. 
Sabemos que para cada $y \in \mathbb{R}^{2}$,

$$
\operatorname{Pr}_{v^{\perp}}\left(\tilde{f}^{q}(y)-y\right)=\operatorname{Pr}_{v^{\perp}}\left(\tilde{f}^{q}\left(x_{0}\right)-x_{0}\right)+\operatorname{Pr}_{v^{\perp}}\left[\left(\tilde{f}^{q}(y)-y\right)-\left(\tilde{f}^{q}\left(x_{0}\right)-x_{0}\right)\right] .
$$

Logo para cada $y \in \mathbb{R}^{2}$, temos dois casos:

(2.6a) Se $\operatorname{Pr}_{v^{\perp}}\left(\tilde{f}^{q}\left(x_{0}\right)-x_{0}\right)>2 M_{0}$, então $\operatorname{Pr}_{v^{\perp}}\left(\tilde{f}^{q}(y)-y\right)>2 M_{0}-M_{0}=M_{0}$.

(2.6b) Se $\operatorname{Pr}_{v^{\perp}}\left(\tilde{f}^{q}\left(x_{0}\right)-x_{0}\right)<-2 M_{0}$, então $\operatorname{Pr}_{v^{\perp}}\left(\tilde{f}^{q}(y)-y\right)<-2 M_{0}+M_{0}=-M_{0}$.

Vejamos o caso (2.6a):

Como $\tilde{f}^{q n}(y)-y=\sum_{i=1}^{n} \tilde{f}^{q}\left(\tilde{f}^{(i-1) q}(y)\right)-\tilde{f}^{(i-1) q}(y)$, para cada $n \in \mathbb{N}$ então,

$$
\operatorname{Pr}_{v^{\perp}}\left(\tilde{f}^{q n}(y)-y\right)=\sum_{i=1}^{n} \operatorname{Pr}_{v^{\perp}}\left[\tilde{f}^{q}\left(\tilde{f}^{(i-1) q}(y)\right)-\tilde{f}^{(i-1) q}(y)\right]>n M_{0} .
$$

Logo, para cada $n \in \mathbb{N}$ e $y \in \mathbb{R}^{2}, \operatorname{Pr}_{v^{\perp}}\left[\frac{\tilde{f}^{q n}(y)-y}{n}\right]>M_{0}$.

Portanto,

$$
\operatorname{Pr}_{v^{\perp}}\left(\rho_{p}\left(\tilde{f}^{q}\right)\right) \subset\left(M_{0},+\infty\right)
$$

Isto contradiz o fato que $0 \in \operatorname{Pr}_{v^{\perp}}(\rho(\tilde{f}))$. Com efeito, pelas propriedades do conjunto de rotação e de (2.7), temos

$$
\begin{array}{lll}
\operatorname{Pr}_{v^{\perp}}(\rho(\tilde{f})) \subset\left(M_{0} / q,+\infty\right), & \text { se } & q \in \mathbb{Z}_{*}^{+}, \\
\operatorname{Pr}_{v^{\perp}}(\rho(\tilde{f})) \subset\left(-\infty, M_{0} / q\right), & \text { se } & q \in \mathbb{Z}_{*}^{-}
\end{array}
$$

Analogamente temos contradição, no caso (2.6b). 


\subsection{Lema de Atkinson}

Nesta seção, enunciamos o conhecido Lema de Atkinson, que será de grande importância na demonstração da Proposição 4.10, do Capítulo 4.

Teorema 2.56 ([Atk76]). Sejam $(X, \mathcal{B}, \mu)$ um espaço de probabilidade, não atômico e $T$ : $X \rightarrow X$ um automorfismo ergódico em relação à $\mu$. Considere $\phi: X \rightarrow \mathbb{R}$ uma função integrável e $\int \phi d \mu=0$. Então para cada $B \in \mathcal{B}$ e cada $\epsilon>0$,

$$
\mu\left(\bigcup_{n \in \mathbb{N}} B \cap T^{-n}(B) \cap\left\{x \in X:\left|\sum_{i=0}^{n-1} \phi\left(T^{i}(x)\right)\right|<\epsilon\right\}\right)=\mu(B) .
$$

Como consequência do teorema anterior, temos o seguinte resultado.

Corolário 2.57 (Lema de Atkinson). Sejam $X$ um espaço métrico separável, $f: X \rightarrow X$ um homeomorfismo e $\mu$ uma medida de probabilidade, não atômica, ergódica e f-invariante. Se $\phi: X \rightarrow \mathbb{R}$ é uma função integrável tal que $\int \phi d \mu=0$, então para $\mu$-q.t. $x \in X$, existe uma sequência crescente $\left(n_{i}\right)_{i \in \mathbb{N}}$ de inteiros positivos, tal que

$$
f^{n_{i}}(x) \rightarrow x \quad e \quad \sum_{k=0}^{n_{i}-1}\left(\phi_{1} \circ f^{k}\right)(x) \rightarrow 0, \quad \text { quando } \quad i \rightarrow \infty .
$$

Demonstração. Basta mostrar que o conjunto $E_{i}$ de todos os $x \in X$, para os quais existe $n \in \mathbb{N}$, tal que

$$
f^{n}(x) \in B_{1 / i}(x) \quad e \quad\left|\sum_{k=0}^{n-1} \phi\left(f^{k}(x)\right)\right|<\frac{1}{i}
$$

tem medida total para cada $i \in \mathbb{N}$.

Suponhamos o contrário, isto é, $\mu\left(X \backslash E_{i}\right)>0$, para algum $i \in \mathbb{N}$. Como $X$ é separável, existe uma cobertura infinita enumerável de $X$, por bolas de radio $\frac{1}{2 i}$. Logo existe $x \in X$ tal que $\mu\left(B_{1 / 2 i}(x) \backslash E_{i}\right)>0$. Mas o Teorema 2.56, aplicado a $B=\left(B_{1 / 2 i}(x) \backslash E_{i}\right)$ e $\epsilon=\frac{1}{i}$, implica que existem $n \in \mathbb{N}$ e $x^{\prime} \in B$ tais que

$$
f^{n}\left(x^{\prime}\right) \in B \quad e \quad\left|\sum_{k=0}^{n-1} \phi\left(f^{k}\left(x^{\prime}\right)\right)\right|<\frac{1}{i} .
$$

Em particular, $f^{n}\left(x^{\prime}\right) \in B_{1 / i}\left(x^{\prime}\right)$, logo pela definição de $E_{i}$, concluímos que $x^{\prime} \in E_{i}$, o que é uma contradição. 


\section{Capítulo 3}

\section{Grupo Abeliano de homeomorfismos de $\mathbb{T}^{2}$}

Neste capítulo, consideramos um semigrupo Abeliano $\mathcal{G}$ finitamente gerado por $s$ homeomorfismos homotópicos à identidade de $\mathbb{T}^{2}, f_{1}, \ldots, f_{s}$, pela operação de composição e $\tilde{\mathcal{G}}$, $\tilde{f}_{1}, \ldots \tilde{f}_{s}$, respectivos levantamentos.

Uma razão para estudar grupos Abelianos de homeomorfismos homotópicos à identidade, vem do seguinte corolário. Para uma demonstração, ver ([Par14], Corolário 2.2).

Corolário 3.1. Em qualquer variedade de dimensão 2, exceto o anel aberto, levantamentos dados pela homotopia de homeomorfismos homotópicos à identidade que comutam, também comutam.

Nossa intenção com este capítulo é buscar entender o quanto da teoria de rotação existente para homeomorfismos do toro pode se estender ao estudo dos semigrupos Abelianos. Introduziremos um novo conceito de conjunto de rotação para esta situação e estudamos algumas propriedades preliminares.

\subsection{Conjunto de rotação do semigrupo}

Definição 3.2. Dado um semigrupo Abeliano $\mathcal{G}$ e um conjunto de geradores $B=\left\{f_{1}, \ldots, f_{s}\right\}$, com levantamentos respectivos $\tilde{\mathcal{G}}$ e $\tilde{B}=\left\{\tilde{f}_{1}, \ldots, \tilde{f}_{s}\right\}$, definimos o conjunto de rotação do semigrupo, denotado por $\rho(\tilde{\mathcal{G}}, \tilde{B})$ como o conjunto de pontos $v \in \mathbb{R}^{2}$ tais que para cada $j=1, \ldots, s$, existem sequências $\left(r_{i}^{j}\right)_{i \in \mathbb{N}} \in \mathbb{N} e\left(x_{i}\right)_{i \in \mathbb{N}} \in \mathbb{R}^{2}$ com $N_{i}=\sum_{j=1}^{s} r_{i}^{j} \rightarrow \infty e$

$$
v=\lim _{i \rightarrow \infty} \frac{\tilde{f}_{1}^{1} \cdots \tilde{f}_{s}^{s}\left(x_{i}\right)-x_{i}}{N_{i}}
$$


Quando for implícito o conjunto de geradores $\tilde{B}$, vamos simplesmente denotar $\rho(\tilde{\mathcal{G}}, \tilde{B})$ por $\rho(\tilde{\mathcal{G}})$.

\section{Propriedades}

Consideremos o semigrupo $\tilde{\mathcal{G}}$, gerado pelo conjunto $\tilde{B}=\{\tilde{f}, \tilde{g}\}$. Sejam $p \in \mathbb{N}$ e $q \in \mathbb{Z}^{2}$. Denotemos o semigrupo $\tilde{\mathcal{G}}^{p}-q=<\tilde{f}^{p}-q, \tilde{g}^{p}-q>$. Definimos o conjunto $\rho\left(\tilde{\mathcal{G}}^{p}-q\right)$ como o conjunto dos $v \in \mathbb{R}^{2}$, tais que existem sequências $\left(n_{i}\right)_{i \in \mathbb{N}},\left(m_{i}\right)_{i \in \mathbb{N}} \in \mathbb{N}$ e $\left(x_{i}\right)_{i \in \mathbb{N}} \in \mathbb{R}^{2}$, com $N_{i}=n_{i}+m_{i} \rightarrow \infty$, quando $i \rightarrow \infty, \mathrm{e}$

$$
v=\lim _{i \rightarrow \infty} \frac{\left(\left(\tilde{f}^{p}-q\right)^{n_{i}}\left(\tilde{g}^{p}-q\right)^{m_{i}}\right)\left(x_{i}\right)-x_{i}}{N_{i}} .
$$

Vejamos a seguinte propriedade do conjunto de rotação do semigrupo $\tilde{\mathcal{G}}$, similar à do conjunto de rotação de $\tilde{f}$.

Propriedade 3.3. Para cada $p \in \mathbb{N}$ e cada $q \in \mathbb{Z}^{2}$,

$$
\rho\left(\tilde{\mathcal{G}}^{p}-q\right)=p \rho(\tilde{\mathcal{G}})-q
$$

Demonstração.

i. Se $v=p w-q$, com $w \in \rho(\tilde{\mathcal{G}})$, então existem sequências $\left(n_{i}\right)_{i \in \mathbb{N}}, \quad\left(m_{i}\right)_{i \in \mathbb{N}} \in \mathbb{N}$ e $\left(x_{i}\right)_{i \in \mathbb{N}} \in \mathbb{R}^{2}$, tais que $N_{i}=n_{i}+m_{i} \rightarrow \infty$ e

$$
v=p \lim _{i \rightarrow \infty} \frac{\tilde{f}^{n_{i}} \tilde{g}^{m_{i}}\left(x_{i}\right)-x_{i}}{N_{i}}-q
$$

Sabemos que existem naturais $r_{1}$ e $r_{2}$ com $0 \leqslant r_{1}, r_{2} \leqslant p-1$, tais que $n_{i}=s_{i} p+r_{1}$ e $m_{i}=l_{i} p+r_{2}$, com $s_{i}=\left\lfloor\frac{p}{n_{i}}\right\rfloor$, e $l_{i}=\left\lfloor\frac{p}{m_{i}}\right\rfloor$, onde $\lfloor\cdot\rfloor$ denota a parte inteira. Assim se $M_{i}=s_{i}+l_{i}$, então $N_{i}=p M_{i}+\left(r_{1}+r_{2}\right)$,

$$
\lim _{i \rightarrow \infty} \frac{p M_{i}}{N_{i}}=1 \quad \text { e } \quad \lim _{i \rightarrow \infty} \frac{\tilde{f}^{r_{1}} \tilde{g}^{r_{2}}\left(x_{i}\right)-x_{i}}{N_{i}}=0 .
$$

Então,

$$
\begin{aligned}
v & =p \lim _{i \rightarrow \infty} \frac{\tilde{f}^{s_{i}} p \tilde{g}^{l_{i} p}\left(\tilde{f}^{r_{1}} \tilde{g}^{r_{2}}\left(x_{i}\right)\right)-x_{i}}{N_{i}}-q \\
& =\lim _{i \rightarrow \infty} \frac{p M_{i}}{N_{i}} \frac{\left(\tilde{f}^{s_{i}} \tilde{g}^{l_{i}}\right)^{p}\left(z_{i}\right)-z_{i}-M_{i} q}{M_{i}},
\end{aligned}
$$

$\operatorname{com} z_{i}=\tilde{f}^{r_{1}} \tilde{g}^{r_{2}}\left(x_{i}\right)$. 
Logo,

$$
v=\lim _{i \rightarrow \infty} \frac{\left(\left(\tilde{f}^{p}-q\right)^{s_{i}}\left(\tilde{g}^{p}-q\right)^{l_{i}}\right)\left(z_{i}\right)-z_{i}}{M_{i}} .
$$

Portanto $v \in \rho\left(\tilde{\mathcal{G}}^{p}-q\right)$ e $p \rho(\tilde{\mathcal{G}})-q \subset \rho\left(\tilde{\mathcal{G}}^{p}-q\right)$.

ii. Se $v \in \rho\left(\tilde{\mathcal{G}}^{p}-q\right)$ então,

$$
\begin{aligned}
v & =\lim _{i \rightarrow \infty} p \frac{\left(\left(\tilde{f}^{p}-q\right)^{n_{i}}\left(\tilde{g}^{p}-q\right)^{m_{i}}\right)\left(x_{i}\right)-x_{i}}{p N_{i}} \\
& =\lim _{i \rightarrow \infty} p \frac{\left(\tilde{f}^{n_{i} p} \tilde{g}^{m_{i} p}\right)\left(x_{i}\right)-x_{i}-N_{i} q}{p N_{i}} \\
& =p v^{\prime}-q,
\end{aligned}
$$

$\operatorname{com} v^{\prime} \in \rho(\tilde{\mathcal{G}})$. Portanto $v \in p \rho(\tilde{\mathcal{G}})-q$ e $\rho\left(\tilde{\mathcal{G}}^{p}-q\right) \subset p \rho(\tilde{\mathcal{G}})-q$.

Observação 3.4. Dado que o semigrupo $\tilde{\mathcal{G}}=<\tilde{f}_{1}, \ldots, \tilde{f}_{s}>$ é Abeliano, podemos ver por indução que para cada $n, k_{j} \in \mathbb{N}, j=1, \ldots, s$,

i) $\left(\tilde{f}_{1}^{k_{1}} \cdots \tilde{f}_{s}^{k_{s}}\right)^{n}=\tilde{f}_{1}^{k_{1} n} \cdots \tilde{f}_{s}^{k_{s} n}$.

ii) $\tilde{f}_{1}^{k_{1}} \cdots \tilde{f}_{s}^{k_{s}}=\left[\tilde{f}_{1}^{k_{1}} \tilde{f}_{2}^{k_{2}} \cdots \tilde{f}_{s}^{k_{s}}-\tilde{f}_{2}^{k_{2}} \cdots \tilde{f}_{s}^{k_{s}}\right]+\cdots+\left[\tilde{f}_{s-1}^{k_{s-1}} \tilde{f}_{s}^{k_{s}}-\tilde{f}_{s}^{k_{s}}\right]+\tilde{f}_{s}^{k_{s}}$.

Propriedade 3.5. Para cada $k_{j} \in \mathbb{N}, j=1, \ldots, s$, se $k=\sum_{j=1}^{s} k_{j} \neq 0$ então,

$$
\frac{1}{k} \rho\left(\tilde{f}_{1}^{k_{1}} \cdots \tilde{f}_{s}^{k_{s}}\right) \subset \rho(\tilde{\mathcal{G}})
$$

Demonstração. Seja $v \in \rho\left(\tilde{f}_{1}^{k_{1}} \cdots \tilde{f}_{s}^{k_{s}}\right)$. Então existem sequências $\left(x_{l}\right)_{l \in \mathbb{N}} \in \mathbb{R}^{2}$ e $\left(n_{l}\right)_{l \in \mathbb{N}} \in \mathbb{N}$ tais que $n_{l} \rightarrow \infty$, quando $l \rightarrow \infty$, e

$$
v=\lim _{l \rightarrow \infty} \frac{\left(\left(\tilde{f}_{1}^{k_{1}} \cdots \tilde{f}_{s}^{k_{s}}\right)^{n_{l}}\right)\left(x_{l}\right)-x_{l}}{n_{l}}
$$

Pela Observação 3.4. i),

$$
\frac{\left(\left(\tilde{f}_{1}^{k_{1}} \cdots \tilde{f}_{s}^{k_{s}}\right)^{n_{l}}\right)\left(x_{l}\right)-x_{l}}{n_{l}}=\frac{\tilde{f}_{1}^{k_{1} n_{l}} \cdots \tilde{f}_{s}^{k_{s} n_{l}}\left(x_{l}\right)-x_{l}}{n_{l}} .
$$

Logo, existe uma subsequência de (3.1) convergente, tal que

$$
\frac{1}{\left(\sum_{j=1}^{s} k_{j}\right)} v=\lim _{i \rightarrow \infty} \frac{\left(\tilde{f}_{1}^{k_{1} n_{i}} \cdots \tilde{f}_{s}^{k_{s} n_{i}}\right)\left(x_{i}\right)-x_{i}}{\sum_{j=1}^{s}\left(k_{j} n_{i}\right)} \in \rho(\tilde{\mathcal{G}}) .
$$


Denotemos o fecho convexo de $\bigcup_{j=1}^{s} \rho\left(\tilde{f}_{j}\right)$ por $R$, isto é,

$$
R=\left\{v \in \mathbb{R}^{2} ; v=\sum_{j=1}^{s} a_{j} v_{j}, \text { onde } a_{j} \in[0,1], \sum_{j=1}^{s} a_{j}=1 \text { e } v_{j} \in \rho\left(\tilde{f}_{j}\right) \text {, para } j=1, \ldots, s\right\} .
$$

Proposição 3.6. Consideremos o conjunto R, definido acima. Então,

$$
\rho(\tilde{\mathcal{G}}) \subset R
$$

Demonstração. Se $v \in \rho(\tilde{\mathcal{G}})$, então existem sequências $\left(x_{l}\right)_{l \in \mathbb{N}} \in \mathbb{R}^{2}$ e $\left(r_{l}^{j}\right)_{l \in \mathbb{N}} \in \mathbb{N}$, para cada $j=1, \ldots, s$, tais que se $l \rightarrow \infty$, então $\sum_{j=1}^{s} r_{l}^{j}=N_{l} \rightarrow \infty$ e

$$
\lim _{l \rightarrow \infty} \frac{\tilde{f}_{1}^{r_{l}^{1}} \cdots \tilde{f}_{s}^{r_{l}^{s}}\left(x_{l}\right)-x_{l}}{N_{l}}=v .
$$

Pela Observação 3.4. ii),

$$
\begin{aligned}
\frac{\tilde{f}_{1}^{r_{l}^{1}} \cdots \tilde{f}_{s}^{r_{l}^{s}}\left(x_{l}\right)-x_{l}}{N_{l}} & =\frac{r_{l}^{1}}{N_{l}}\left[\frac{\tilde{f}_{1}^{r_{l}^{1}}\left(\tilde{f}_{2}^{r_{l}^{2}} \cdots \tilde{f}_{s}^{r_{l}^{s}}\left(x_{l}\right)\right)-\left(\tilde{f}_{2}^{r_{l}^{2}} \cdots \tilde{f}_{s}^{r_{l}^{s}}\left(x_{l}\right)\right)}{r_{l}^{1}}\right]+\cdots+ \\
& +\frac{r_{l}^{s-1}}{N_{l}}\left[\frac{\tilde{f}_{s-1}^{r_{l}^{s-1}}\left(\tilde{f}_{s}^{r_{l}^{s}}\left(x_{l}\right)\right)-\left(\tilde{f}_{s}^{r_{l}^{s}}\left(x_{l}\right)\right)}{r_{l}^{s-1}}\right]+\frac{r_{l}^{s}}{N_{l}}\left[\frac{\tilde{f}_{s}^{r_{l}^{s}}\left(x_{l}\right)-\left(x_{l}\right)}{r_{l}^{s}}\right] .
\end{aligned}
$$

Denotemos para cada $j=1, \ldots, s$, a sequência $\left(\sigma_{l}^{j}\right)_{l \in \mathbb{N}}$ dada por

$$
\sigma_{l}^{j}=\frac{\tilde{f}_{j}^{r_{l}}\left(z_{l}\right)-\left(z_{l}\right)}{r_{l}^{j}}, \text { onde }\left(z_{l}\right)_{l \in \mathbb{N}} \in \mathbb{R}^{2}
$$

Pela Propriedade 2.15, para cada $j=1, \ldots, s$, existe alguma subsequência convergente $\left(\sigma_{i}^{j}\right)_{i \in \mathbb{N}}$ tal que, para cada $j=1, \ldots, s, \sigma_{i}^{j} \rightarrow v_{j} \in \rho\left(\tilde{f}_{j}\right)$, se $i \rightarrow \infty$.

Seja $a_{l}^{j}=\frac{r_{l}^{j}}{N_{l}} \in[0,1]$, para $l \in \mathbb{N}$, então para cada $j=1, \ldots, s$, existe alguma subsequência convergente $\left(a_{i}^{j}\right)_{i \in \mathbb{N}}$ tal que, $a_{i}^{j} \rightarrow a^{j} \in[0,1]$, se $i \rightarrow \infty$ e $\sum_{j=1}^{s} a^{j}=1$.

Logo,

$$
v=\lim _{i \rightarrow \infty} \frac{\tilde{f}_{1}^{r_{i}^{1}} \cdots \tilde{f}_{s}^{r_{i}^{s}}\left(x_{i}\right)-x_{i}}{N_{i}}=\sum_{j=1}^{s} a^{j} v_{j}
$$

Portanto, $v \in R$. 
Propriedade 3.7. Temos que

$$
\operatorname{Conv}(\rho(\tilde{\mathcal{G}}))=R .
$$

Demonstração. Da Propriedade 3.5 temos que $\rho\left(\tilde{f}_{j}\right) \subset \rho(\tilde{\mathcal{G}})$, para cada $j=1, \ldots, s$. Logo $\bigcup_{i=1}^{s} \rho\left(\tilde{f}_{i}\right) \subset \rho(\tilde{\mathcal{G}})$. Portanto,

$$
R=\operatorname{Conv}\left(\bigcup_{i=1}^{s} \rho\left(\tilde{f}_{i}\right)\right) \subset \operatorname{Conv}(\rho(\tilde{\mathcal{G}}))
$$

Mas pela Proposicão 3.6,

$$
\operatorname{Conv}(\rho(\tilde{\mathcal{G}})) \subset R
$$

Exemplo 3.8. O conjunto de rotação do semigrupo não precisa ser convexo.

Consideremos o semigrupo $\tilde{\mathcal{G}}=<\tilde{f}, \tilde{f}^{k}>$, para $k \in \mathbb{Z}$.

Então,

$$
\begin{aligned}
& \rho(\tilde{\mathcal{G}}) \subset \bigcup_{t \in[1, k]}\{t \rho(\tilde{f})\}, \quad \text { se } k \geq 1, \quad \text { e } \\
& \rho(\tilde{\mathcal{G}}) \subset \bigcup_{t \in[k, 1]}\{t \rho(\tilde{f})\}, \quad \text { se } k \leqslant 1 .
\end{aligned}
$$

Em particular, sejam $k=-1$ e $\rho(\tilde{f})=[0,1]^{2}$. Então, temos que o ponto $p=\left(-\frac{1}{2}, \frac{1}{2}\right) \in R$, mas $p \notin \rho(\tilde{\mathcal{G}})$, pois

$$
\rho(\tilde{\mathcal{G}}) \subset \bigcup_{t \in[-1,1]}\{t \rho(\tilde{f})\} \subset R
$$

e p $\bigcup_{t \in[-1,1]}\{t \rho(\tilde{f})\}$, que não é convexo.

Proposição 3.9. Consideremos o semigrupo $\tilde{\mathcal{G}}=<\tilde{f}, \tilde{g}>$. Se $\rho(\tilde{f})=\{p\}$ então, $R \subset \rho(\tilde{G})$. Demonstração. Consideremos as sequências $\left(y_{l}\right)_{l \in \mathbb{N}} \in \mathbb{R}^{2}$ e $\left(s_{l}\right)_{l \in \mathbb{N}} \in \mathbb{N}$, com $s_{l} \rightarrow \infty$, tais que

$$
\lim _{l \rightarrow \infty} \frac{\tilde{g}^{s_{l}}\left(y_{l}\right)-y_{l}}{s_{l}}=q
$$

para algum $q \in \rho(\tilde{g})$.

Dado $a \in[0,1]$, existem uma sequência $\left(r_{l}\right)_{l \in \mathbb{N}} \in \mathbb{N}$, tal que $a_{l}=\frac{r_{l}}{N_{l}} \in[0,1]$, com $N_{l}=r_{l}+s_{l}$ e uma subsequência convergente $\left(a_{k}\right)_{k \in \mathbb{N}}$, onde $a_{k} \rightarrow a \in[0,1]$.

Pela Propriedade 2.15, temos que dadas quaisquer sequências $\left(z_{l}\right)_{l \in \mathbb{N}} \in \mathbb{R}^{2}$ e $\left(r_{l}\right)_{l \in \mathbb{N}} \in \mathbb{N}$, a sequência $\sigma_{l}(f)=\frac{\tilde{f}^{r} l\left(z_{l}\right)-z_{l}}{r_{l}}$ possui uma subsequência convergente $\sigma_{k}(f)$. Da hipótese segue que $\lim _{k \rightarrow \infty} \sigma_{k}(f)=p$. 
Denotemos $z_{k}=\tilde{g}^{s_{k}}\left(y_{k}\right)$ então,

$$
\frac{\tilde{f}^{r_{k}} \tilde{g}^{s_{k}}\left(y_{k}\right)-y_{k}}{N_{k}}=a_{k}\left[\frac{\tilde{f}^{r_{k}}\left(z_{k}\right)-z_{k}}{r_{k}}\right]+\left(1-a_{k}\right)\left[\frac{\tilde{g}^{s_{k}}\left(y_{k}\right)-y_{k}}{s_{k}}\right] .
$$

Logo, se $v=a p+(1-a) q \in R$ então,

$$
v=\lim _{k \rightarrow \infty} \frac{\tilde{f}^{r_{k}} \tilde{g}^{s_{k}}\left(y_{k}\right)-y_{k}}{N_{k}} .
$$

Portanto, $v \in \rho(G)$. 


\subsection{Grupo anular}

Nesta seção seguimos as ideias em [Ben13].

Definimos o deslocamento de $\tilde{\mathcal{G}}$, por

$$
\operatorname{Desl}(\tilde{\mathcal{G}})=\sup _{\substack{\tilde{g} \in \tilde{\mathcal{E}} \\ x \in \mathbb{R}^{2}}}\|\tilde{g}(x)-x\| .
$$

Se $v \in \mathbb{R}_{*}^{2}$, definimos o deslocamento de $\tilde{G}$ na direção de $v$, por

$$
\operatorname{Desl}_{v}(\tilde{\mathcal{G}})=\sup _{\substack{\tilde{g} \in \tilde{\mathcal{G}} \\ x \in \mathbb{R}^{2}}}\left|\operatorname{Pr}_{v}(\tilde{g}(x)-x)\right|
$$

Definição 3.10. Seja $v \in \mathbb{Z}_{*}^{2}$. Dizemos que $\tilde{\mathcal{G}}$ é $v$-anular, se o deslocamento de $\tilde{\mathcal{G}}$ na direção de $v^{\perp}$ é limitado.

Um semigrupo $\mathcal{G}=<f_{1}, \ldots, f_{s}>$ é v-anular, se existem levantamentos $\tilde{f}_{j}$ de $f_{j}$, para cada $j=1, \ldots, s$, tais que $\tilde{\mathcal{G}}=<\tilde{f}_{1}, \ldots, \tilde{f}_{s}>$ é v-anular. E dizemos que $\mathcal{G}$ é anular, se existe $v \in \mathbb{Z}_{*}^{2}$ tal que $\mathcal{G}$ é v-anular.

Temos:

Proposição 3.11. O semigrupo Abeliano $\tilde{\mathcal{G}}=<\tilde{f}_{1}, \ldots, \tilde{f}_{s}>$ é anular se, e somente se, $\tilde{f}_{j}$ é anular, para cada $j=1, \ldots, s$.

Demonstração. Se $\tilde{\mathcal{G}}$ é anular, segue da definição que, em particular, cada $\tilde{f}_{j}$ é anular.

Suponhamos que $\tilde{f}_{j}$ é anular, para cada $j=1, \ldots, s$. Então, para cada $j=1, \ldots, s$, existe $M_{j}>0$, tal que $\left|\operatorname{Pr}_{v}\left(\tilde{f}_{j}^{n}(x)-x\right)\right| \leqslant M_{j}$, para cada $n \in \mathbb{Z}$.

Dado qualquer $\tilde{g} \in \tilde{\mathcal{G}}$, existe $k_{j}$, para $j=1, \ldots, s$, tal que $\tilde{g}=\tilde{f}_{1}^{k_{1}} \cdots \tilde{f}_{s}^{k_{s}}$.

Logo,

$$
\begin{aligned}
\left|\operatorname{Pr}_{v^{\perp}}(\tilde{g}(x)-x)\right| \leqslant & \left|\operatorname{Pr}_{v^{\perp}}\left(\tilde{f}_{1}^{k_{1}} \tilde{f}_{2}^{k_{2}} \cdots \tilde{f}_{s}^{k_{s}}(x)-\tilde{f}_{2}^{k_{2}} \cdots \tilde{f}_{s}^{k_{s}}(x)\right)\right|+ \\
& +\cdots+\left|\operatorname{Pr}_{v^{\perp}}\left(\tilde{f}_{s}^{k_{s}}(x)-x\right)\right| \leqslant M_{1}+\cdots+M_{s}=M .
\end{aligned}
$$

\subsection{Resultados recentes}

Consideremos um semigrupo Abeliano $\mathcal{G}$ gerado por $f_{1}, \ldots, f_{s} \in \operatorname{hom}_{0}\left(\mathbb{T}^{2}\right)$. Denotemos

$$
\operatorname{Fix}(\mathcal{G})=\bigcap_{1 \leqslant j \leqslant s} \operatorname{Fix}\left(f_{j}\right)
$$


Dizemos que um semigrupo $\mathcal{G}$ é irrotacional, se $\rho(\tilde{\mathcal{G}})=0$.

Recentemente, aparece o primeiro trabalho conhecido sobre conjuntos de rotação de grupos Abelianos de homeomorfismos no toro homotópicos à identidade, conservativos (isto é, homeomorfismos que preservam uma medida boreliana de probabilidade de suporte total), na tese de doutorado de M. Benayon [Ben13].

No teorema principal é mostrada uma generalização do Teorema 2.32, enunciado no capítulo anterior ([KT14a], Teorema A), para o caso de grupos Abelianos irrotacionais $(\rho(\tilde{\mathcal{G}})=0)$, finitamente gerados. Mais precisamente, temos:

Teorema 3.12. Seja $\mathcal{G}$ um grupo Abeliano de homeomorfismos de $\mathbb{T}^{2}$ homotópicos à identidade, finitamente gerado por homeomorfismos conservativos. Se $\mathcal{G}$ é irrotacional e $\tilde{\mathcal{G}}$ é seu levantamento irrotacional, vale uma das seguintes afirmações:

1. O conjunto Fix $(\tilde{\mathcal{G}})$ é totalmente essencial.

2. Toda órbita por $\tilde{\mathcal{G}}$ é limitada.

3. O grupo $\tilde{\mathcal{G}}$ é anular.

E ainda temos os seguintes resultados.

Teorema 3.13. Seja $\mathcal{G}$ um grupo Abeliano finitamente gerado por difeomorfismos de $\mathbb{T}^{2}$, de classe $\mathbb{C}^{1}$, homotópicos à identidade e conservativos. Se $\mathcal{G}$ é irrotacional, então $F i x(\mathcal{G}) \neq \emptyset$.

Teorema 3.14. Seja $\mathcal{G}$ um grupo Abeliano de homeomorfismos de $\mathbb{T}^{2}$ homotópicos à identidade. Suponha que $\mathcal{G}$ é finitamente gerado e que todo gerador de $\mathcal{G}$ possui um levantamento que contém a origem no seu conjunto de rotação. Se $\mathcal{G}$ contém algum elemento conservativo possuindo um levantamento com conjunto de rotação sendo um segmento não degenerado de inclinação racional, então $\mathcal{G}$ é anular.

Teorema 3.15. Sejam $f$ e g difeomorfismos de $\mathbb{T}^{2}$ de classe $\mathbb{C}^{1}$ homotópicos à identidade e que comutam. Se $f$ é irrotacional e conservativo e $0 \in$ int $\rho(\tilde{g})$ para algum levantamento $\tilde{g}$ de $g$, então Fix $(f, g) \neq \emptyset$.

O seguinte resultado ([Ben13], Proposição 3.1) é fundamental no próximo capítulo, para a demonstração da Proposição 4.10 .

Proposição 3.16. Sejam $f, h \in \operatorname{hom}_{0}\left(\mathbb{T}^{2}\right)$ que comutam e $\tilde{f}, \tilde{h}$ respectivos levantamentos. Suponhamos também que $f$ é não errante. Se $\tilde{f}$ é $v$-anular para algum $v \in \mathbb{Z}_{*}^{2}$, então $\operatorname{Desv}_{v^{\perp}}(\tilde{h})<\infty$ ou $\operatorname{Desv}(\tilde{f})<\infty$. 


\section{Capítulo 4}

\section{Restrições aos conjuntos de rotação}

\subsection{Motivação do problema}

O objetivo principal desta tese é o seguinte:

Queremos saber se dados dois conjuntos $K_{1}, K_{2} \in \mathbb{R}^{2}$ convexos e compactos, existem dois homeomorfismos $f, h \in \operatorname{hom}_{0}\left(\mathbb{T}^{2}\right)$ que comutam, cujos conjuntos de rotação sejam $K_{1}$ e $K_{2}$, respectivamente.

Como vimos no Capítulo 2, dado um homeomorfismo do toro, homotópico à identidade, podemos classificar seu conjunto de rotação segundo sua forma em três tipos: ou um ponto, ou um segmento compacto, ou um conjunto com interior não vazio. Vamos nos restringir ao estudo dos conjuntos de rotação nos seguintes casos, onde é conhecida a existência de homeomorfismos com estes conjuntos de rotação.

\section{Casos 4.1.}

1. Um único ponto.

2. Um segmento de reta com inclinação racional com pontos racionais.

3. Um polígono com vértices racionais.

A seguir, daremos exemplos de levantamentos de homeomorfismos $f, h \in \operatorname{hom}_{0}\left(\mathbb{T}^{2}\right)$, para os quais sabemos que $f$ e $h$ comutam. Para isso, consideremos $\rho(\tilde{f})$ em cada caso, como segue.

\section{Um único ponto}

Dados quaisquer pares de pontos no plano, sempre existem duas pseudo-rotações que comutam, sendo esses pontos seus conjuntos de rotação. 
Exemplo 4.2. Consideremos as rotações $f, h$ com levantamentos $\tilde{f}, \tilde{h}$, definidos por:

$$
\begin{aligned}
& \tilde{f}(x, y)=(x, y)+\left(z_{1}, z_{2}\right), \\
& \tilde{h}(x, y)=(x, y)+\left(w_{1}, w_{2}\right),
\end{aligned}
$$

para quaisquer $\left(z_{1}, z_{2}\right),\left(w_{1}, w_{2}\right) \in \mathbb{R}^{2}$.

Então,

$$
\begin{aligned}
& \rho(\tilde{f})=\left\{\left(z_{1}, z_{2}\right)\right\}, \\
& \rho(\tilde{h})=\left\{\left(w_{1}, w_{2}\right)\right\},
\end{aligned}
$$

$e$

$$
(\tilde{f} \circ \tilde{h})(x, y)=(x, y)+\left(z_{1}, z_{2}\right)+\left(w_{1}, w_{2}\right)=(\tilde{h} \circ \tilde{f})(x, y)
$$

Vejamos que qualquer rotação racional sempre comuta com algum homeomorfismo cujo conjunto de rotação é qualquer uma das possibilidades dos Casos 4.1.

Exemplo 4.3. Consideremos qualquer $g \in \operatorname{hom}_{0}\left(\mathbb{T}^{2}\right)$, $\tilde{g}$ um levantamento de $g, v=(p, q) \in$ $\mathbb{Q}^{2}$ e $k \in \mathbb{Z}$, tal que $k v \in \mathbb{Z}^{2}$. Definimos os levantamentos $\tilde{f}, \tilde{h}$ da seguinte forma:

$$
\begin{aligned}
& \tilde{f}(z)=z+v, \\
& \tilde{h}(z)=\frac{1}{k} \tilde{g}(k z) .
\end{aligned}
$$

Então, $h \in \operatorname{hom}_{0}\left(\mathbb{T}^{2}\right)$. Podemos ver por indução que $\tilde{h}^{n}(z)=\frac{1}{k} \tilde{g}^{n}(k z)$. Desta maneira,

$$
k\left[\tilde{h}^{n}(z)-z\right]=\tilde{g}^{n}(k z)-z=\left(\tilde{g}^{n}(k z)-k z\right)+(k z-z) .
$$

Consequentemente,

$$
k \rho_{p}(\tilde{h})=\rho_{p}(\tilde{g})
$$

E ainda, das propriedades do conjunto de rotação obtemos,

$$
\rho\left(\tilde{h}^{k}\right)=\rho(\tilde{g}) .
$$

Portanto, $\rho(\tilde{f})=\{v\}$ e, se $\rho(\tilde{g})$ é algum dos conjuntos dos Casos 4.1, temos que $\rho(\tilde{h})$ também é. 
Já que $\tilde{g}(z+k v)=\tilde{g}(z)+k v$, concluímos que

$$
(\tilde{h} \circ \tilde{f})(z)=\frac{1}{k} \tilde{g}^{n}(k z+k v)=\tilde{h}(z)+v=(\tilde{f} \circ \tilde{h})(z) . \diamond
$$

\section{Um segmento de reta}

Dados dois segmentos de reta com a mesma inclinação racional, passando pela origem, sempre existem homeomorfismos que comutam, com esses conjuntos de rotação.

Exemplo 4.4. Consideremos $\varphi: \mathbb{R} \rightarrow[a, b]$ e $\psi: \mathbb{R} \rightarrow[c, d]$ funções contínuas, sobrejetoras, periódicas e de período um, onde $a<b$ e $c<d$. Definimos os levantamentos $\tilde{f}, \tilde{h}$ por:

$$
\begin{aligned}
& \tilde{f}(x, y)=(x, y)+(0, \varphi(x)), \\
& \tilde{h}(x, y)=(x, y)+(0, \psi(x)) .
\end{aligned}
$$

Então,

$$
\begin{aligned}
& \rho(\tilde{f})=\{0\} \times[a, b], \\
& \rho(\tilde{h})=\{0\} \times[c, d],
\end{aligned}
$$

$e$

$$
(\tilde{f} \circ \tilde{h})(x, y)=(x, y)+(0, \varphi(x)+\psi(x))=(\tilde{h} \circ \tilde{f})(x, y) .
$$

Exemplo 4.5. Em particular, se considerarmos no exemplo anterior a função $\psi(x)=\gamma \notin \mathbb{Q}$, teremos casos de homeomorfismos $f, h$ que comutam, tais que $\rho(\tilde{f})$ é um segmento de reta com inclinação racional e $\rho(\tilde{h})$ é um ponto com uma coordenada racional e a outra irracional.

\section{Um polígono com vértices racionais}

Se $f \in \operatorname{hom}_{0}\left(\mathbb{T}^{2}\right)$, nesse caso, os exemplos mais simples de homeomorfismos que comutam com $f$ são suas potências. Vejamos a continuação.

Exemplo 4.6. Consideremos qualquer $f \in \operatorname{hom}_{0}\left(\mathbb{T}^{2}\right), \tilde{f}$ um levantamento de $f$ tal que $\rho(\tilde{f})$ seja um polígono com vértices racionais $K$ e seja $h=f^{p}$, para qualquer $p \in \mathbb{N}$. Então $f$ e $h$ comutam e pelas propriedades do conjunto de rotação, $\rho(h)=p K$.

Neste exemplo temos homeomorfismos que comutam cujos conjuntos de rotação tem interior não vazio. 
Exemplo 4.7. Observemos que no Exemplo 4.3, $\rho(\tilde{g})$ pode ser um polígono com vértices racionais. Portanto g comuta com qualquer rotação racional.

Questão 5. Dado um homeomorfismo $f$ cujo conjunto de rotação é um segmento de reta com inclinação racional $\theta$, contendo mais de um ponto racional, como podem ser os conjuntos de rotação dos homeomorfismos que comutam com $f$ ? Podem ser dos seguintes tipos?

\section{Casos 4.8.}

1. Um segmento de reta, não degenerado, com inclinação $\beta \neq \theta$.

2. Com interior não vazio.

O Teorema B, que veremos neste capítulo, da resposta negativa nos Casos 4.8.

\subsection{Demonstração do teorema}

\section{Lema auxiliar}

Podemos ver que o seguinte lema está contido na Proposição 3.16, ([Ben13], Proposição 3.1). Incluiremos sua demonstração por completude.

Denotemos as translações em $\mathbb{R}^{2}, T_{1}(z)=z+(1,0)$ e $T_{2}(z)=z+(0,1)$, e as projeções na primeira variável $\operatorname{Pr}_{1}$ e na segunda variável $\operatorname{Pr}_{2}$.

Lema 4.9. Sejam $f, h \in \operatorname{hom}_{0}\left(\mathbb{T}^{2}\right)$ que comutam e $\tilde{f}, \tilde{h}$ respectivos levantamentos. Se $\tilde{f} e ́$ $(0,1)$-anular e $\operatorname{Desv}_{(1,0)}(\tilde{h})$ é ilimitado, então existe um conjunto $E \subset \mathbb{R}^{2}$, com as seguintes propriedades:

i.) O conjunto $E$ é aberto, $\tilde{f}$-invariante e $T_{2}(E)=E$.

ii.) As componentes conexas de E têm diâmetro uniformemente limitado.

iii.) Para cada $x \in \mathbb{R}^{2}$, existe $u \in \mathbb{Z}^{2}$ tal que $(x+u) \in E$, isto é, $\pi(E)=\mathbb{T}^{2}$.

Demonstração. i.) Consideremos o semiplano

$$
H=\left\{(x, y) \in \mathbb{R}^{2} ; x \geq 0\right\}
$$

E definimos o conjunto

$$
B=\overline{\bigcup_{n \in \mathbb{Z}} \tilde{f}^{n}(H)}
$$


Então, $\tilde{f}(B)=B$ e $T_{2}(B)=B$. Como $\tilde{f}$ é $(0,1)$-anular, existe $M>0$, tal que $\operatorname{Desl}_{(1,0)}(\tilde{f}) \leqslant M$. Logo,

$$
B \subset\left\{(x, y) \in \mathbb{R}^{2} ; x \geq-M\right\}
$$

Denotemos com $\Delta=\sup _{z \in \mathbb{R}^{2}}\|\tilde{f}(z)-z\| \mathrm{e}$

$$
A^{\prime}=B \backslash\left[T_{1}^{k}(\operatorname{int}(B))\right],
$$

onde $k>2 M+\Delta+1$. Note que $\tilde{f}\left(A^{\prime}\right)=A^{\prime}$.

Então, o conjunto

$$
A^{\prime \prime}=\left\{(x, y) \in \mathbb{R}^{2} ; M \leqslant x \leqslant M+\Delta+1\right\} \subset A^{\prime} .
$$

Seja $A$ uma componente conexa de $A^{\prime}$, tal que $A^{\prime \prime} \subset A$. Vemos que $\tilde{f}(A) \cap A \neq \emptyset$, assim $\tilde{f}(A)=A$. Além disso, $T_{2}(A)=A$, $\operatorname{diam}\left(\operatorname{Pr}_{1}(A)\right) \leqslant k+2 M$ e $\left(T_{2} \circ \tilde{h}^{n}\right)(A)=\tilde{h}^{n}(A)$, para cada $n \in \mathbb{Z}$.

Consideremos $l \in \mathbb{N}$ com $l>2(k+2 M+1)$, de maneira que o conjunto

$$
F=\left\{(x, y) \in \mathbb{R}^{2} ; k+M \leqslant x \leqslant l-M\right\}
$$

seja tal que, diam $\left(\operatorname{Pr}_{1}(F)\right)>k+2 M+2$. Veja a Figura 4.1.

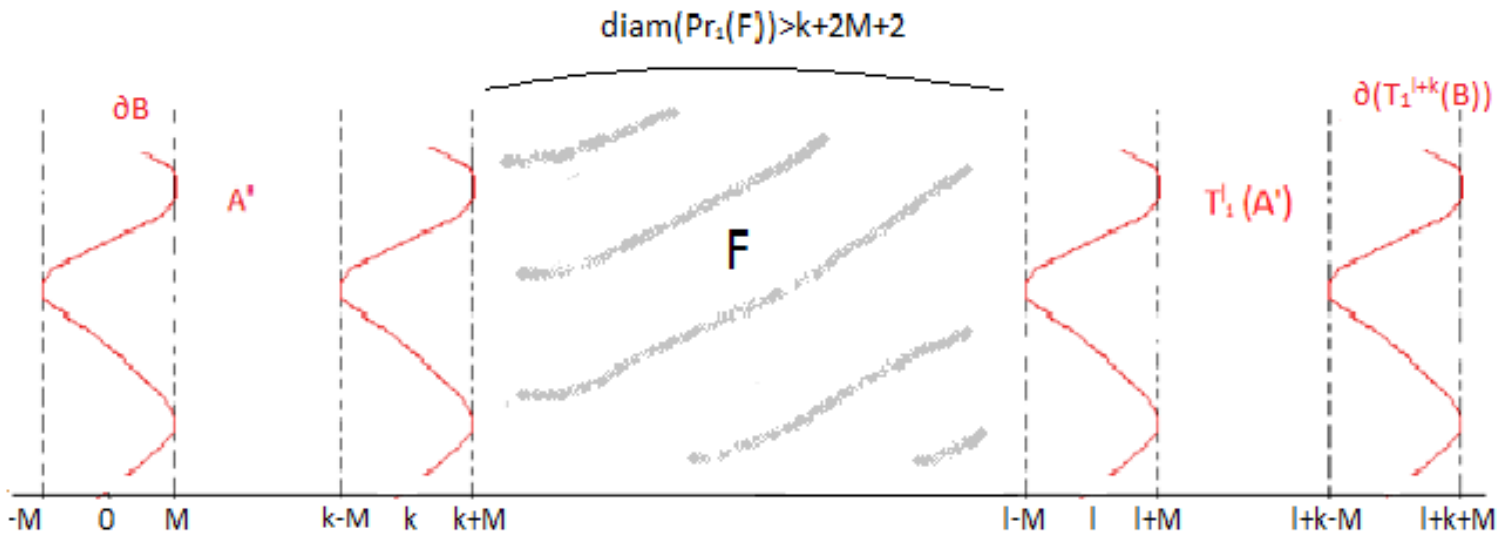

Figura 4.1: Os conjuntos $A^{\prime}$ e F.

Consequentemente,

$$
\operatorname{diam}\left[\operatorname{Pr}_{1}\left(B \backslash \operatorname{int}\left(T_{1}^{l+k}(B)\right)\right)\right] \leqslant l+k+2 M .
$$

Portanto,

$$
\operatorname{diam}\left[\operatorname{Pr}_{1}\left(B \backslash \operatorname{int}\left(T_{1}^{2 l+k}(B)\right)\right)\right] \leqslant 2(l+k+2 M) .
$$


Dado que, $\operatorname{Desv}_{(1,0)}(\tilde{h})$ é ilimitado, existem $n \in \mathbb{Z}, z_{1}, z_{2} \in \mathbb{R}^{2}$ tais que,

$$
\left|\operatorname{Pr}_{1}\left[\left(\tilde{h}^{n}\left(z_{1}\right)-z_{1}\right)-\left(\tilde{h}^{n}\left(z_{2}\right)-z_{2}\right)\right]\right|>2(l+k+2 M) .
$$

Já que diam $\left(\operatorname{Pr}_{1}(A)\right) \leqslant k+2 M$, em particular, temos que se $z_{1}, z_{2} \in A$, então

$$
\left|\operatorname{Pr}_{1}\left[\tilde{h}^{n}\left(z_{1}\right)-\tilde{h}^{n}\left(z_{2}\right)\right]\right|>2 l+k+2 M .
$$

Isto permite supor que $\tilde{h}^{n}\left(z_{1}\right) \in A$ e $\tilde{h}^{n}\left(z_{2}\right) \in T_{1}^{l}(A)$ (eventualmente trocando $\tilde{h}^{n}$ por $\tilde{h}^{n}+T_{1}^{j}$, para algum $j \in \mathbb{Z}$ ). Veja a Figura 4.2.

Definimos,

$$
E=\left[T_{1}^{k}(\operatorname{int}(B)) \backslash T_{1}^{l}(B)\right] \backslash \tilde{h}^{n}(A) .
$$

Vemos que $E$ é aberto, $\tilde{f}$-invariante e $T_{2}(E)=E$, pois $B$ e $\tilde{h}^{n}(A)$ também são.

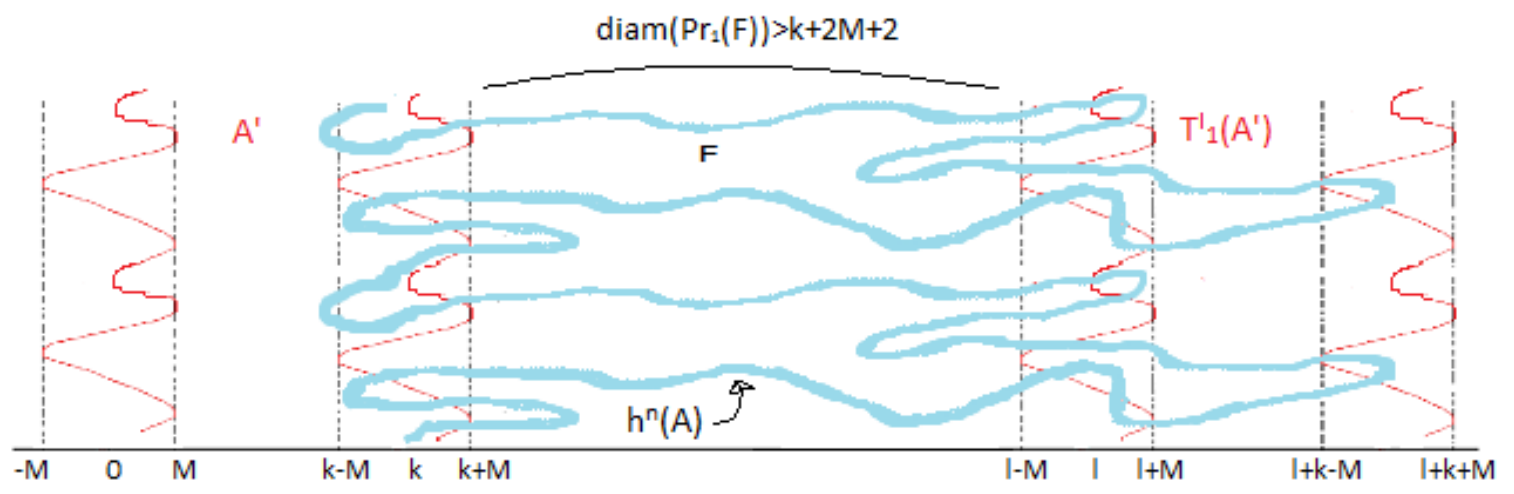

Figura 4.2: $O$ conjunto $h^{n}(A)$.

ii.) Vejamos que existe $M_{0}>0$ tal que, $\operatorname{diam}(U) \leqslant M_{0}$, sendo $U$ qualquer componente conexa de $E$.

Dado que $E$ está contido em $T_{1}^{k}(\operatorname{int}(B)) \backslash T_{1}^{l}(B)$, concluímos que

$$
\operatorname{diam}\left(\operatorname{Pr}_{1}(E)\right) \leqslant(l+M)-(k-M)=M_{1} .
$$

Seja $\gamma:[0,1] \rightarrow \mathbb{R}^{2}$ um caminho contido em $\tilde{h}^{n}(A)$, ligando $A$ a $T_{1}^{l}(A)$. Como $\left(T_{2}^{j} \circ\right.$ $\left.\tilde{h}^{n}\right)(A)=\tilde{h}^{n}(A)$, para cada $n, j \in \mathbb{Z}$, temos que o caminho $T_{2}^{j}(\gamma)$ também está contido em $\tilde{h}^{n}(A)$, para cada $j \in \mathbb{Z}$, e liga $A$ a $T_{1}^{l}(A)$. Como $\gamma$ é compacto, existem $a, b \in \mathbb{R}$ tais que $a \leqslant \operatorname{Pr}_{2}(z) \leqslant b$, para cada $z \in \gamma$. Então, para qualquer componente conexa $U$ de $E$, $\operatorname{diam}\left(\operatorname{Pr}_{2}(U)\right) \leqslant|b-a+1|$.

Desta maneira, consideremos $M_{2}=|b-a+1|$ e $M_{0}=\sqrt{M_{1}^{2}+M_{2}^{2}}$. 
iii.) Vejamos que para cada $z \in \mathbb{R}^{2}$, existe $u \in \mathbb{Z}^{2}$ tal que $z+u \in E$.

Suponhamos por absurdo que existe $z \in \mathbb{R}^{2}$ tal que $\left(z+\mathbb{Z}^{2}\right) \cap E=\emptyset$. Porém existe $u \in \mathbb{Z}^{2}$ tal que $w=z+u \in F$, logo $w \in \tilde{h}^{n}(A)$. Como $\operatorname{diam}\left(\operatorname{Pr}_{1}(F)\right)>k+2 M+2$, podemos escolher $u$ de tal forma que $T_{1}^{j}(w) \in\left(F \cap \tilde{h}^{n}(A)\right)$, para $j=0, \ldots, k+2 M+1 . \operatorname{Logo} \tilde{h}^{-n}\left(T_{1}^{j}(w)\right) \in A$, são $k+2 M+2$ transladados de um ponto em $A$. Isto contradiz que $\operatorname{diam}\left(\operatorname{Pr}_{1}(A)\right) \leqslant k+2 M$.

\subsubsection{Resultado principal}

Na proposição a seguir, denotemos por $L=\{t(0,1) ; t \in \mathbb{R}\}$.

Proposição 4.10. Sejam $f, h \in \operatorname{hom}_{0}\left(\mathbb{T}^{2}\right)$ que comutam e $\tilde{f}, \tilde{h}$ respectivos levantamentos. Se $\tilde{f}$ é $(0,1)$-anular, então $\operatorname{Desv}_{(1,0)}(\tilde{h})<\infty$ ou $\operatorname{Desv}(\tilde{f})<\infty$.

Demonstração. Suponhamos que $\operatorname{Desv}_{(1,0)}(\tilde{h})$ é ilimitado. Então, existe um conjunto $E$ com as propriedades descritas no Lema 4.9 .

Dado que as componentes conexas de $E$ tem diâmetro uniformemente limitado, consideremos $M_{0}>0$ tal que, para toda componente conexa $U$ de $E$, diam $(U)<M_{0}$.

Afirmação 1. Existem $U$ uma componente conexa de $E, \bar{n} \in \mathbb{Z}^{+}$e $\bar{m} \in \mathbb{Z}$, tais que

$$
\left\|\tilde{f}^{k \bar{n}}(x)-x-k(0, \bar{m})\right\| \leqslant M_{0},
$$

para cada $k \in \mathbb{N}$ e cada $x \in U$.

Com efeito, pela Observação 2.53, podemos supor que $\rho(\tilde{f}) \subset L$, isto é, ou $\rho(\tilde{f})=$ $\{0\} \times[a, b]$, com $a<b$, ou $\rho(\tilde{f})=\{(0, a)\}$, onde $a, b \in \mathbb{R}$. Em qualquer caso, existe pelo menos um ponto extremal de $\rho(\tilde{f})$ da forma $(0, a)$.

Do Teorema 2.21 sabemos que existe $\mu \in \mathcal{M}_{e}(f)$ tal que para $\mu$-q.t $x \in \mathbb{T}^{2}$,

$$
\rho(\mu, f)=\int \phi d \mu=(0, a)
$$

onde $\phi: \mathbb{T}^{2} \rightarrow \mathbb{R}^{2}$ é a função deslocamento definida por $\phi(x)=\tilde{f}(\tilde{x})-(\tilde{x})$, para $\tilde{x} \in \pi^{-1}(x)$. Logo, da Proposição 2.20, segue que para $\mu$-q.t $x \in \mathbb{T}^{2}$ e cada $\tilde{x} \in \pi^{-1}(x)$,

$$
\lim _{n \rightarrow \infty} \frac{\tilde{f}^{n}(\tilde{x})-\tilde{x}}{n}=(0, a) .
$$

Consideremos a projeção na primeira variável $\operatorname{Pr}_{1}: \mathbb{R}^{2} \rightarrow \mathbb{R}$ e a função $\phi_{1}: \mathbb{T}^{2} \rightarrow \mathbb{R}$, definida por $\phi_{1}(x)=\left(\operatorname{Pr}_{1}(\phi)(x)\right)$. 
Então,

$$
\int \phi_{1} d \mu=0
$$

Pelo Lema de Atkinson (Corolário 2.57), aplicado à função $\phi_{1}$, temos que para $\mu$-q.t $x \in \mathbb{T}^{2}$, existe uma sequência crescente de inteiros positivos $\left(n_{i}\right)_{i \in \mathbb{N}}$, tal que

$$
f^{n_{i}}(x) \rightarrow x \quad e \quad \sum_{i=0}^{n_{i}-1}\left(\phi_{1} \circ f^{k}\right)(x) \rightarrow 0, \quad \text { quando } \quad i \rightarrow \infty .
$$

Isto é, para cada $\mu$-q.t $x \in \mathbb{T}^{2}$ e $\tilde{x} \in \pi^{-1}(x)$,

$$
\operatorname{Pr}_{1}\left[\tilde{f}^{n_{i}}(\tilde{x})-\tilde{x}\right] \rightarrow 0, \quad \text { quando } \quad i \rightarrow \infty
$$

Consideremos $x \in \mathbb{T}^{2}$ satisfazendo (4.1) e $\tilde{x}=\pi^{-1}(x)$, então pela propriedade iii.) de $E$, existe $u \in \mathbb{Z}^{2}$ tal que $\tilde{x}+u=w \in U$, para alguma componente conexa $U$ de $E$. Assim, existem sequências $\left(n_{i}\right)_{i \in \mathbb{N}} \in \mathbb{Z}^{+}$e $\left(m_{i}\right)_{i \in \mathbb{N}} \in \mathbb{Z}$, tais que $\tilde{f}^{n_{i}}(w)-T_{2}^{m_{i}}(w) \rightarrow 0$, quando $i \rightarrow \infty$.

Como $U$ é aberto, seja $\epsilon>0$ tal que $B_{\epsilon}(w) \subset U$. Então, existe $i_{0} \in \mathbb{N}$ tal que para todo $i>i_{0}, \tilde{f}^{n_{i}}(w) \in T_{2}^{m_{i}}\left(B_{\epsilon}(w)\right) \subset T_{2}^{m_{i}}(U)$. Em particular, para algum $\bar{m} \in \mathbb{Z}$, existe $\bar{n} \in \mathbb{Z}^{+}$tal que $\tilde{f}^{\bar{n}}(U) \cap T_{2}^{\bar{m}}(U) \neq \emptyset$. Pela propriedade $i$.) de $E$, temos que $T_{2}^{m}(U)$ também é uma componente conexa de $E$, para cada $m \in \mathbb{Z}$. Como $E$ é $\tilde{f}$-invariante, $\tilde{f}$ permuta as componentes conexas de $E$. Então $\tilde{f}^{\bar{n}}(U)=T_{2}^{\bar{m}}(U)$. Por indução, vemos que $\tilde{f}^{k \bar{n}}(U)=$ $T_{2}^{k \bar{m}}(U)$, para cada $k \in \mathbb{N}$.

Como $M_{0}>\operatorname{diam}(U)$ então,

$$
\left\|\tilde{f}^{k \bar{n}}(x)-x-k(0, \bar{m})\right\| \leqslant M_{0}, \quad \text { para cada } x \in U \text { e } k \in \mathbb{N} . \diamond
$$

Consideremos $D$ um domínio fundamental que contém $w$.

Afirmação 2. Existe uma cobertura finita $C=\bigcup_{j=1}^{s}\left\{U_{j}\right\}$ de $D$, onde cada $U_{j}$ é um aberto, conexo, tal que para algum $u_{j} \in \mathbb{Z}^{2}, U_{j}+u_{j}$ é componente conexa de $E$.

Com efeito, pela propriedade $i i i$.) de $E$, para cada ponto $y \in D$, existe um $u_{*} \in \mathbb{Z}^{2}$, tal que $\left(y+u_{*}\right) \in U_{y}^{*}$, para alguma componente conexa $U_{y}^{*}$ de $E$.

Sejam $U^{\prime}=U_{y}^{*}-u_{*}$ e $C^{\prime}=\bigcup_{y \in D}\left\{U_{y}^{\prime}\right\}$. Pelas propriedades de $E$, o conjunto $C^{\prime}$ é uma cobertura de $D$ por abertos, conexos, tais que $U^{\prime}+u_{*}$ é uma componente conexa de $E$. Além disso, $C^{\prime}$ contém o conjunto $U=U_{w}^{*}$.

Como $D$ é compacto podemos escolher uma subcobertura finita, isto é, existe uma sub- 
família finita de $C^{\prime}$ que também cobre $D$, denotemos-a por

$$
C=\bigcup_{j=1}^{s}\left\{U_{j}\right\}, \text { onde }\left\{U_{j}\right\} \subset C^{\prime} \text {, para cada } j=1, \ldots, s \text {. } \diamond
$$

Seja $U_{j}^{*}=U_{j}+u_{j}$ componente conexa de $E$, para algum $u_{j} \in \mathbb{Z}^{2}$. Como $\tilde{f}$ permuta as componentes conexas de $E$, então temos que para cada $n \in \mathbb{Z}, \tilde{f}^{n}\left(U_{j}\right)=\tilde{f}^{n}\left(U_{j}^{*}\right)-u_{j}$ é um transladado de uma componente conexa de $E$. Então, pela propriedade ii.) de $E$, $\operatorname{diam}\left(\tilde{f}^{n}\left(U_{j}\right)\right)<M_{0}$, para cada $n \in \mathbb{Z}$ e cada $j=1, \ldots, s$.

Podemos supor que $\left\{U_{1}\right\}=\{U\} \subset C$, onde $U$ é o aberto da Afirmação 1 (caso contrário consideramos a cobertura finita $\{U\} \cup C)$. Como $D$ é conexo, então sem perda de generalidade, podemos enumerar os abertos $U_{j}, j=1, \ldots, s$, da cobertura $C$, de tal forma que cada $U_{j}$ intercepte algum dos $U_{l}$, para $1 \leqslant l<j$, isto é, considerar $U_{j}$ tal que,

$$
U_{j} \cap\left[\bigcup_{1 \leqslant l<j} U_{l}\right] \neq \emptyset, \text { para cada } j=2, \ldots, s .
$$

Afirmação 3. Existem $M>0$ e $p \in \mathbb{Q}^{2}$ tais que, $\left\|\tilde{f}^{k}(y)-y-k p\right\| \leqslant M$, para cada $y \in D$ e cada $k \in \mathbb{N}$.

Com efeito, vejamos por indução sobre $j$ que $\left\|\tilde{f}^{k \bar{n}}(y)-y-k(0, \bar{m})\right\| \leqslant(2 j-1) M_{0}$, para cada $y \in U_{j}, \quad j=1, \ldots, s$, e cada $k \in \mathbb{N}$, onde $\bar{n}$ e $\bar{m}$ são como na Afirmação 1 .

i. O caso $j=1$ é a Afirmação 1.

ii. Suponhamos válido para cada $U_{l}$, com $1 \leqslant l \leqslant j$, e vejamos o caso $j+1$ :

Por (4.2), temos que $U_{j+1} \cap U_{l} \neq \emptyset$, para algum $U_{l}$ com $1 \leqslant l \leqslant j$. Então, $\tilde{f}^{\bar{n}}\left(U_{j+1}\right) \cap$ $\tilde{f}^{\bar{n}}\left(U_{l}\right) \neq \emptyset$. Por indução vemos que, $\tilde{f}^{k \bar{n}}\left(U_{j+1}\right) \cap \tilde{f}^{k \bar{n}}\left(U_{l}\right) \neq \emptyset$, para cada $k \in \mathbb{N}$.

Como $\operatorname{diam}\left(\tilde{f}^{n}\left(U_{j}\right)\right)<M_{0}$, para cada $n \in \mathbb{Z}$ e cada $j=1, \ldots, s$, então para cada $y \in U_{j+1}$, cada $k \in \mathbb{N}$ e algum $x \in U_{j+1} \cap U_{l}$ temos,

$$
\begin{aligned}
\left\|\tilde{f}^{k \bar{n}}(y)-y-k(0, \bar{m})\right\| & \leqslant\left\|\tilde{f}^{k \bar{n}}(y)-\tilde{f}^{k \bar{n}}(x)\right\|+\left\|\tilde{f}^{k \bar{n}}(x)-x-k(0, \bar{m})\right\|+\|x-y\| \\
& \leqslant M_{0}+(2 l-1) M_{0}+M_{0} \leqslant(2 j+1) M_{0}=[2(j+1)-1] M_{0} .
\end{aligned}
$$

Seja $M_{1}=(2 s-1) M_{0}$, então $\left\|\tilde{f}^{k \bar{n}}(y)-y-k(0, \bar{m})\right\|<M_{1}$, para cada $y \in D$ e cada $k \in N$.

Denotemos por $p=\frac{1}{\bar{n}}(0, \bar{m}) \in \mathbb{Q}^{2}$. Sabemos que dado $k \in \mathbb{N}$, existem $t, r \in \mathbb{N}$, tais que $k=t \bar{n}+r$, onde $0 \leqslant r \leqslant \bar{n}$. 
Logo, para cada $y \in D$ e cada $k \in \mathbb{N}$,

$$
\begin{aligned}
\left\|\tilde{f}^{k}(y)-y-k p\right\| & =\left\|\tilde{f}^{t \bar{n}+r}(y)-y-(t \bar{n}+r) p\right\| \\
& \leqslant\left\|\tilde{f}^{r}\left(\tilde{f}^{t \bar{n}}(y)\right)-\tilde{f}^{t \bar{n}}(y)\right\|+\left\|\tilde{f}^{t \bar{n}}(y)-y-t(0, \bar{m})\right\|+\|r p\| \\
& \leqslant n_{i} \sup _{z \in \mathbb{R}^{2}}\|\tilde{f}(z)-z\|+M_{1}+r\|p\|=M . \diamond
\end{aligned}
$$

Dado $z \in \mathbb{R}^{2}$, seja $u \in \mathbb{Z}^{2}$ tal que $z+u=y \in D$.

Então,

$$
\left\|\tilde{f}^{k}(z)-z-k p\right\|=\left\|\tilde{f}^{k}(z+u)-(z+u)-k p\right\|=\left\|\tilde{f}^{k}(y)-y-k p\right\| \leqslant M
$$

Portanto, dados $z \in \mathbb{R}^{2}$ e $k \in \mathbb{N}$, existem $M>0$ e $p \in \mathbb{Q}^{2}$, tais que

$$
\left\|\tilde{f}^{k}(z)-z-k p\right\| \leqslant M
$$

Isto implica que $\operatorname{Desv}(\tilde{f})<\infty$.

Consideremos agora o caso geral, para qualquer $v \in \mathbb{Z}_{*}^{2}$.

Teorema A. Sejam $f, h \in \operatorname{hom}_{0}\left(\mathbb{T}^{2}\right)$ que comutam e $\tilde{f}, \tilde{h}$ respectivos levantamentos. Se $\tilde{f}$ é v-anular, para algum $v \in \mathbb{Z}_{*}^{2}$, então $\operatorname{Desv}_{v^{\perp}}(\tilde{h})<\infty$ ou $\operatorname{Desv}(\tilde{f})<\infty$.

Demonstração. Segue da Observação 2.51, que se $\tilde{f}$ é $v$-anular então $A \tilde{f} A^{-1}$ é $(0,1)$-anular. Desta forma os homeomorfismos $A \tilde{f} A^{-1}$ e $A \tilde{h} A^{-1}$ satisfazem as mesmas hipóteses da Proposição 4.10 .

\subsection{Consequências}

Consideremos $v \in \mathbb{Z}_{*}^{2}$ e a reta $L=\{t v ; t \in \mathbb{R}\}$.

Teorema B. Se $\rho(\tilde{f})$ é um segmento da reta $L$, com pontos racionais e $\rho(\tilde{h})$ é qualquer um dos seguintes casos:

1. Tem interior não vazio.

2. Um segmento de reta não degenerado, numa direção não paralela à $v$.

3. Um ponto contido na reta $L$, sendo $\tilde{h}$ não $v$-anular.

Então, em cada caso $\tilde{f}$ e $\tilde{h}$ não comutam. 
Demonstração. Suponhamos por contradição que $\tilde{f}$ e $\tilde{h}$ comutam. Pelo Teorema 2.40 ([Dav13], Teorema A), sabemos que $\tilde{f}^{k}$ é $v$-anular, para algum $k \in \mathbb{N}$. Denotemos $\tilde{g}=\tilde{f}^{k}$, então $\tilde{g}$ e $\tilde{h}$ comutam.

Afirmação. Em qualquer um dos casos para $\rho(\tilde{h}), \operatorname{Desv}_{v^{\perp}}(\tilde{h})$ é ilimitado.

Com efeito, o caso 1. segue da Observação 2.52. O caso 2. segue da Proposição 2.54. E o caso 3., da Proposição 2.55.

Logo, o Teorema A implica que $\operatorname{Desv}(\tilde{g})<\infty$. Portanto, $\tilde{g}$ é uma pseudo-rotação. Isto contradiz que $\rho(\tilde{f})$ é um segmento da reta $L$, não degenerado.

Corolário 4.11. Se $\rho(\tilde{f})$ é um segmento da reta L, contendo pontos racionais e $\tilde{h}$ é transitivo, então $\tilde{f}$ e $\tilde{h}$ não comutam.

Demonstração. Por [Tal12], sabemos que se $\tilde{h}$ é transitivo, então $0 \in \operatorname{int}(\rho(\tilde{h}))$. O resultado segue do Teorema B.

\section{Grupo anular}

Observação 4.12. O Teorema B implica que se um homeomorfismo $f$ do toro, homotópico à identidade, cujo conjunto de rotação é um segmento de reta não degenerado, contido na reta $L$, comuta com um homeomorfismo h do toro, homotópico à identidade, então seu conjunto de rotação tem interior vazio e temos os seguintes casos:

i. Se $\tilde{h}$ é uma pseudo-rotação contida em L, então $\tilde{h}$ é v-anular.

ii. Se $\rho(\tilde{h})$ é um segmento de reta, não degenerado, então ele deve estar contido numa reta com a mesma direção $v$.

Exemplo 4.13. Suponhamos que $f$ e $h$ comutam. Se $\rho(\tilde{f})=\{0\} \times[a, b]$, com $a<b$ e $\rho(\tilde{h})=(0, \beta)$. Então $\tilde{h}$ é $(0,1)$-anular.

Agora, consideremos como na Seção 3.1, um subgrupo Abeliano $\mathcal{G}$ gerado por $f_{1}, \ldots, f_{s} \in$ $\operatorname{hom}_{0}\left(\mathbb{T}^{2}\right)$ pela operação de composição, e $\tilde{\mathcal{G}}, \tilde{f}_{1}, \ldots, \tilde{f}_{s}$, levantamentos respectivos. Denote$\operatorname{mos}$ a reta $L=\{t v ; t \in \mathbb{R}\}$, para algum $v \in \mathbb{Z}_{*}^{2}$.

Lembremos que um grupo $\tilde{\mathcal{G}}$ é $v$-anular, se o deslocamento de $\tilde{\mathcal{G}}$ na direção de $v^{\perp}$ é limitado, para algum $v \in \mathbb{Z}_{*}^{2}$. O seguinte resultado é uma versão do Teorema 3.14 ([Ben13], Teorema C), sem a hipótese de ser $f$ conservativo. 
Teorema C. Seja $\mathcal{G}$ como acima. Suponhamos que todo gerador $f_{i}$ possui um levantamento $\tilde{f}_{i}$, tal que $0 \in \operatorname{Pr}_{v^{\perp}}\left(\rho\left(\tilde{f}_{i}\right)\right)$, e que existe algum elemento $f \in \mathcal{G}$, tal que $\rho(\tilde{f})$ é um segmento não degenerado contido na reta $L$. Então, $\mathcal{G}$ é anular.

Demonstração. Segue das Proposições 3.11, 2.55 e do Teorema B. 


\section{Bibliografia}

[Atk76] G. Atkinson. Recurrence of cocycles and random walks. J. London Math. Soc. 13, no.2, pp. 486-488, 1976. 24

[AZ02] S. Addas-Zanata. On the existence of a new type of periodic and quasi-periodic orbits for twist maps of the torus. Nonlinearity 15, pp. 1399-1416, 2002. 9

[AZ15] S. Addas-Zanata. Uniform bounds for diffeomorphisms of the torus and a conjecture of Boyland. J. London Math. Soc. 91, no.2, pp. 537-553, 2015. 20

[AZTG14] S. Addas-Zanata, F. Tal e B. A. Garcia. Dynamics of homeomorphisms of the torus homotopic to Dehn twists. Ergod. Th. Dynam. Sys. 34, no.2, pp 409-422, 2014. 20

[BCH14] P. Boyland, A. De Carvalho e T. Hall. New rotation sets in a family of torus homeomorphisms. arXiv:1410.7727v1, 2014. 14

[BCJR09] F. Beguin, S. Crovisier, T. Jager e F. Le Roux. Denjoy constructions for fibered homeomorphisms of the torus. Trans. Amer. Math. Soc. 361, no. 11, pp. 58515883, 2009. 17

[BCRP04] F. Beguin, S. Crovisier, F. Le Roux e A. Patou. Pseudo-rotations of the closed annulus: variation on a theorem of J. Kwapisz. Nonlinearity 17, pp. 1427-1453, 2004. 17

[Beg07] F. Beguin. Ensembles de rotations des homeomorphismes du tore $\mathbb{T}^{2}$. Notes de classe, 2007. 13

[Ben13] M. Benayon. Sobre grupos abelianos irrotacionais de homeomorfismos do toro. Tese - Universidade Federal Fluminense, 2013. 2, 21, 31, 32, 36, 43

[Boy09] P. Boyland. Transitivity of surface dynamics lifted to Abelian covers. Ergod. Th. Dynam. Sys.,pp 1417-1449, 2009. 20

[BR07] F. Beguin e F. Le Roux. Dynamique topologique sur les surfaces. Notes de classe, 2007. 7

[CT15] P. Le Calvez e F. Tal. Forcing theory for transverse trajectories of surface homeomorphisms. arXiv:1503.09127v1, 2015. 2, 14, 17, 20

[Dav13] P. Davalos. On annular maps of the torus and sublinear diffusion. arXiv:1311.0046, 2013. 18, 43

[Den32] A. Denjoy. Sur les courbes definies par les equations differentielles a la surface du tore. J. Math. Pures Appl. 9, 11, pp. 333-376, 1932. 1, 5 
[Doe97] E. Doeff. Rotation measures for homeomorphisms of the torus homotopic to a Dehn twist. Ergod. Th. Dynam. Sys. 17, pp. 575-591, 1997. 9

[Eps66] D. B. A. Epstein. Curves on 2-manifolds and isotopies. Acta Mathematica. 115, pp. $83-107,1966.6$

[FM90] J. Franks e M. Misiurewicz. Rotation sets of toral flows. Proc. Amer. Math. Soc. 109 1, pp. 243-249, 1990. 2, 13

[Fra88] J. Franks. Recurrence and fixed points of surface homeomorphisms. Ergod. Th. Dynam. Sys. 8*, pp. 99-107, 1988. 15

[Fra89] J. Franks. Realizing rotation vectors for torus homeomorphisms. Trans. Amer. Math. Soc. 311, no.1, pp. 107-115, 1989. 19

[Fra95] J. Franks. The rotation set and periodic points for torus homeomorphisms. Dynam. Sys. and Chaos (Aoki, Shiraiwa and Takahashi, eds.), World Scientific, Singapore, pp. 41-48, 1995. 17

[GKT12] N. Guelman, A. Koropecki e F. Tal. Rotation sets with non-empty interior and transitivity in the universal covering. Ergod. Th. Dynam. Sys., pp 1-12, 2012. 20

[GKT14] N. Guelman, A. Koropecki e F. Tal. A characterization of annularity for area preserving toral homeomorphisms. Math. Z. 276, pp. 673-689, 2014. 18

[Jag09a] T. Jager. The concept of bounded mean motion for toral homeomorphisms. Dynam. Sys. 24 3, pp. 277-297, 2009. 17

[Jag09b] T. Jager. Linearization of conservative toral homeomorphisms. Invent. Math. 176, pp. 601-616, 2009. 17

[JZ98] L. Jonker e L. Zhang. Torus homeomorphisms whose rotation sets have empty interior. Ergod. Th. Dynam. Sys. 18, pp. 1173-1185, 1998. 17

[KK08] A. Kocksard e A. Koropecki. Free curves and periodic points for torus homeomorphisms. Ergod. Th. Dynam. Sys. 28, pp. 1895-1915, 2008. 13

[KT12] A. Koropecki e F. Tal. Area preserving irrotational diffeomorphisms of the torus with sublinear diffusion. Proc. of the Amer. Math. Soc. , no. , pp., 2012. 16

[KT14a] A. Koropecki e F. Tal. Bounded and unbounded behavior for area-preserving rational pseudo-rotations. Proc. London Math. Soc. 109, no. 3, pp. 785-822, 2014. $2,16,32$

[KT14b] A. Koropecki e F. Tal. Strictly toral dynamics. Invent math. 196, pp. 339-381, 2014. 19

[Kwa92] J. Kwapisz. Every convex polygon with rational vertices is a rotation set. Ergod. Th. Dynam. Sys. 12, pp. 333-339, 1992. 2, 14

[Kwa95] J. Kwapisz. A toral diffeomorphism with a nonpolygonal rotation set. Nonlinearity 8, pp. 461-476, 1995. 14 
[LM91] J. Llibre e R.S. Mackay. Rotation vectors and entropy for homeomorphisms of the torus isotopic to the identity. Ergod. Th. Dynam. Sys. 11, pp. 115-128, 1991. 1,19

[MZ89] M. Misiurewics e K. Ziemian. Rotation sets for maps of tori. J. London Math. Soc. 40, no.2, pp. 490-506, 1989. 1, 6, 10, 12, 14, 15

[MZ91] M. Misiurewicz e K. Ziemian. Rotation sets and ergodic measures for torus homeomorphisms. Fundamenta Mathematicae 137, pp. 44-52, 1991. 19

[Par14] K. Parkhe. Commuting homeomorphisms with non-commuting lifts. arXiv:1409.6422v2, 2014. 25

[Poi52] H. Poincare. Oeuvres completes. Gauthier-Villars, Paris, 1952. 1, 5

[Pol92] M. Pollicot. Rotation sets for homeomorphisms and homology. Trans. Amer. Math. Soc. 331, no. 2, pp. 881-894, 1992. 6

[Roc70] R. Tyrrell Rockafellar. Convex Analysis. Princeton University Press, 1970. 12

[Sch57] S. Schwartzman. Asymptotic cycles. Ann. Math. 66, no.2, pp. 270-284, 1957. 6

[Tal12] F. Tal. Transitivity and rotation sets with nonempty interior for homeomorphisms of the 2-torus. Proc. Amer. Math. Soc., 140, pp. 3567-3579., 2012. 20, 43

[Tal14] F. Tal. On non-contractible periodic orbits for surface homeomorphisms. arXiv:1307.1664v2, 2014. 16 